\title{
MONOIDAL CATEGORIES OF MODULES OVER QUANTUM AFFINE ALGEBRAS OF TYPE A AND B
}

\author{
MASAKI KASHIWARA, MYUNGHO KIM, AND SE-JIN OH
}

\begin{abstract}
We construct an exact tensor functor from the category $\mathcal{A}$ of finitedimensional graded modules over the quiver Hecke algebra of type $A_{\infty}$ to the category $\mathscr{C}_{B_{n}^{(1)}}$ of finite-dimensional integrable modules over the quantum affine algebra of type $B_{n}^{(1)}$. It factors through the category $\mathcal{T}_{2 n}$, which is a localization of $\mathcal{A}$. As a result, this functor induces a ring isomorphism from the Grothendieck ring of $\mathcal{T}_{2 n}$ (ignoring the gradings) to the Grothendieck ring of a subcategory $\mathscr{C}_{B_{n}^{(1)}}^{0}$ of $\mathscr{C}_{B_{n}^{(1)}}$. Moreover, it induces a bijection between the classes of simple objects. Because the category $\mathcal{T}_{2 n}$ is related to categories $\mathscr{C}_{A_{2 n-1}^{(t)}}^{0}(t=1,2)$ of the quantum affine algebras of type $A_{2 n-1}^{(t)}$, we obtain an interesting connection between those categories of modules over quantum affine algebras of type $A$ and type $B$. Namely, for each $t=1,2$, there exists an isomorphism between the Grothendieck ring of $\mathscr{C}_{A_{2 n-1}^{(t)}}^{0}$ and the Grothendieck ring of $\mathscr{C}_{B_{n}^{(1)}}^{0}$, which induces a bijection between the classes of simple modules.
\end{abstract}

\section{Contents}

Introduction 2

1. Symmetric quiver Hecke algebras and quantum affine algebras 5

1.1. Cartan datum and quantum groups 5

1.2. Quiver Hecke algebras 6

1.3. R-matrices for quiver Hecke algebra 8

1.4. Quantum affine algebras $\quad 10$

1.5. R-matrices for quantum affine algebras $\quad 12$

1.6. Hernandez-Leclerc's subcategory 14

1.7. KLR-type quantum affine Schur-Weyl duality functors 14

2. Quantum affine algebra of type B and duality functors 18

2.1. Quantum affine algebra of type $\mathrm{B}_{n}^{(1)} \quad 18$

2010 Mathematics Subject Classification. 81R50, 16G, 16T25,17B37.

Key words and phrases. Quantum affine algebra, Quiver Hecke algebra, Quantum group, SchurWeyl duality.

The research of M. Kashiwara was supported by Grant-in-Aid for Scientific Research (B) 15H03608, Japan Society for the Promotion of Science.

The research of M. Kim was supported by the National Research Foundation of Korea(NRF) Grant funded by the Korea government(MSIP) (NRF-2017R1C1B2007824).

The research of S.-j. Oh was supported by the National Research Foundation of Korea(NRF) Grant funded by the Korea government(MSIP) (NRF-2016R1C1B1010721). 
2.2. The quiver $\Gamma \quad 20$

2.3. Simple modules over quiver Hecke algebra of type $\mathrm{A}_{\infty} \quad 21$

2.4. Properties of the functor $\mathcal{F} \quad 22$

2.5. Quotients and localizations of the category R-gmod 25

2.6. Factoring through $\mathcal{T}_{N} \quad 27$

3. Relation between quantum affine algebras of type A and type B 35

3.1. Isomorphisms between Grothendieck rings 35

3.2. Images of modules associated with segments 36

$\begin{array}{ll}\text { References } & 37\end{array}$

\section{INTRODUCTION}

Let $\mathfrak{g}$ be a Kac-Moody algebra of affine type and let $U_{q}^{\prime}(\mathfrak{g})$ be the corresponding quantum affine algebra. Since the category $\mathscr{C}_{\mathfrak{g}}$ of finite-dimensional integrable representations of $U_{q}^{\prime}(\mathfrak{g})$ has a rich structure, it has been extensively investigated with various approaches (see for example, $[2,6,8,18,22]$ ). In particular, when $U_{q}^{\prime}(\mathfrak{g})$ is the quantum affine algebra of type $A_{N-1}^{(1)}$, there is a functor, so called the quantum affine Schur-Weyl duality functor, from the category of finite-dimensional modules over the affine Hecke algebra to the category $\mathscr{C}_{A_{N-1}^{(1)}}([3,4,7])$. The KLR-type quantum affine Schur-Weyl duality, which is introduced and developed in [11, 12, 14, 15, 19], is a wide generalization of the quantum affine Schur-Weyl duality. It provides a general procedure to obtain a functor from the category of modules over a symmetric quiver Hecke algebra to the category $\mathscr{C}_{\mathfrak{g}}$ for the quantum affine algebra $U_{q}^{\prime}(\mathfrak{g})$ of arbitrary type. Recall that a symmetric quiver Hecke algebra, also called a symmetric Khovanov-Lauda-Rouquier algebra, is a family of graded algebras associated with a quiver without loops. It is introduced as a generalization of the affine Hecke algebra in the context of categorification of quantum groups $([21,24])$. In the present paper, we consider the quantum affine algebra of type $B_{n}^{(1)}$ and we apply the general procedure of the KLR-type quantum affine Schur-Weyl duality in order to obtain a functor from the category of finite-dimensional graded modules over the symmetric quiver Hecke algebra of type $A_{\infty}$ to the category $\mathscr{C}_{B_{n}^{(1)}}$. As a consequence, we have a close relation between the categories of finite-dimensional integrable modules over quantum affine algebras of type $A$ and that of type $B$.

For a detailed explanation of our results, let us briefly recall the construction of KLR-type quantum affine Schur-Weyl duality functors. Suppose that an index set $J$ and a family $\left\{\left(V_{j}\right)_{X(j)}\right\}_{j \in J}$ of evaluation modules of good modules over $U_{q}^{\prime}(\mathfrak{g})$ are given. Then one can form a quiver $\Gamma^{J}$ such that the set of vertices is $J$ and the set of arrows is given by the order of poles of the normalized $R$-matrices between the modules in the family. Then one obtains a symmetric quiver Hecke algebra $R^{J}$ associated with the quiver $\Gamma^{J}$, and constructs a tensor functor from the category of finite-dimensional graded modules over $R^{J}$ to the category $\mathscr{C}_{\mathfrak{g}}$. We call it the $K L R$-type quantum affine Schur-Weyl duality functor. 
The case when $\mathfrak{g}$ is of type $A_{N-1}^{(1)}$ and the family is given as $\left\{V\left(\varpi_{1}\right)_{q^{2 k}}\right\}_{k \in \mathbb{Z}}$ is thoroughly analyzed in [11]. In this cases, the quiver $\Gamma^{J}$ is of type $A_{\infty}$ with the set of vertices $J=\mathbb{Z}$ and hence the corresponding symmetric quiver Hecke algebra $R^{J}$ is of type $A_{\infty}$. Let $\mathcal{A}$ be the category of finite-dimensional graded modules over the symmetric quiver Hecke algebra of type $A_{\infty}$. One of the main features of this case is that there exists a localization $\mathcal{T}_{N}$ of the category $\mathcal{A}$ such that the duality functor factors through the category $\mathcal{T}_{N}$. Moreover, the category $\mathcal{T}_{N}$, as well as $\mathscr{C}_{A_{N-1}^{(1)}}$, is a rigid tensor category. If we consider the smallest Serre subcategory $\mathscr{C}_{A_{N-1}^{(1)}}^{0}$ of $\mathscr{C}_{A_{N-1}^{(1)}}$ which is stable under taking tensor products and contains a sufficiently large family of fundamental representations in $\mathscr{C}_{A_{N-1}^{(1)}}$ (for the precise definition see, Subsection 1.6), then the duality functor induces a ring isomorphism between the Grothendieck ring $K\left(\mathcal{T}_{N}\right)_{q=1}$ and the Grothendieck ring $K\left(\mathscr{C}_{A_{N-1}^{(1)}}^{0}\right)$ of the category $\mathscr{C}_{A_{N-1}^{(1)}}^{0}$. Here the ring $K\left(\mathcal{T}_{N}\right)_{q=1}$ is obtained from the Grothendieck ring $K\left(\mathcal{T}_{N}\right)$ of the category $\mathcal{T}_{N}$ by ignoring the gradings. Furthermore, the functor induces a bijection between the sets of the classes of simple objects.

In [14], it is shown that the category $\mathcal{T}_{N}$ is related not only to the category $\mathscr{C}_{A_{N-1}^{(1)}}^{0}$ but also to the category $\mathscr{C}_{A_{N-1}^{(2)}}^{0}$ of the twisted quantum affine algebra $U_{q}^{\prime}\left(A_{N-1}^{(2)}\right)$ in a similar fashion. More precisely, there is a dimension-preserving bijection between the fundamental representations in the category $\mathscr{C}_{A_{N-1}^{(1)}}^{0}$ and those in the category $\mathscr{C}_{A_{N-1}^{(2)}}^{0}$ which is induced from the KLR-type quantum affine Schur-Weyl duality functors from the category $\mathcal{A}$ to the category $\mathscr{C}_{A_{N-1}^{(t)}}^{0}(t=1,2)$. Here the KLR-type quantum affine Schur-Weyl duality functor arises from the choice of the family $\left\{V\left(\varpi_{1}\right)_{q^{2 k}}\right\}_{k \in \mathbb{Z}}$ in $\mathscr{C}_{A_{N-1}^{(t)}}^{0}$. Moreover, this duality functor also factors through the category $\mathcal{T}_{N}$ and it induces a ring isomorphism between the Grothendieck rings $K\left(\mathcal{T}_{N}\right)_{q=1}$ and $K\left(\mathscr{C}_{A_{N-1}^{(2)}}^{0}\right)$, which is a bijection on the sets of classes of simple objects. Hence, in conclusion, the three Grothendieck rings $K\left(\mathscr{C}_{A_{N-1}^{(1)}}^{(1)}\right), K\left(\mathscr{C}_{A_{N-1}^{(2)}}^{0}\right)$ and $K\left(\mathcal{T}_{N}\right)_{q=1}$ are isomorphic and those isomorphisms induce bijections between the classes of the simple objects.

The main result of the present paper is that there is an exact tensor functor from the category $\mathcal{T}_{2 n}$ to the category $\mathscr{C}_{B_{n}^{(1)}}^{0}$ and it induces a ring isomorphism between the Grothendieck rings, which is a bijection between the sets of classes of simple objects. An interesting point is that the same category $\mathcal{T}_{2 n}$ plays a role, even we move from the quantum affine algebra of type $A$ to the quantum affine algebra of type $B$. To obtain such a functor, first we present a family $\left\{\left(V_{j}\right)_{X(j)}\right\}_{j \in \mathbb{Z}}$ of $U_{q}^{\prime}\left(B_{n}^{(1)}\right)$-modules, where $V_{j}$ is either $V\left(\varpi_{1}\right)$ or $V\left(\varpi_{n}\right)$ and $X: \mathbb{Z} \rightarrow \mathbb{Q}(q)^{\times}$is a function such that the corresponding quiver $\Gamma$ is of type $A_{\infty}$ (for the precise definition, see Subsection 2.2). Since each module $\left(V_{j}\right)_{X(j)}$ belongs to the category $\mathscr{C}_{B_{n}^{(1)}}^{0}$, the corresponding KLR-type quantum affine Schur-Weyl duality functor $\mathcal{F}$ is a functor from the category $\mathcal{A}$ to the category $\mathscr{C}_{B_{n}^{(1)}}^{0}$. Even though the choice of the family $\left\{\left(V_{j}\right)_{X(j)}\right\}_{j \in \mathbb{Z}}$ is more complicated 
in comparison to the case of the types $A_{N-1}^{(t)}(t=1,2)$, the duality functor $\mathcal{F}$ enjoys several similar properties: Recall that for each pair $a \leq b$ of integers, there is a simple module $L(a, b)$ over the symmetric quiver Hecke algebra of type $A_{\infty}$. Then the functor $\mathcal{F}$ sends the module $L(a, b)$ to zero if and only if $b-a+1>2 n$, and it sends $L(a, b)$ with $b-a+1=2 n$ to the trivial representation of $U_{q}^{\prime}\left(B_{n}^{(1)}\right)$. It follows that the kernel of the functor $\mathcal{F}$ coincides with the ones of the duality functors for type $A_{2 n-1}^{(t)}(t=1,2)$. Moreover, we show that the functor $\mathcal{F}$ factors through the category $\mathcal{T}_{2 n}$ again. To see this, it is enough to show that there exists a family $\left\{c_{i, j}(u, v)\right\}_{i, j \in J}$ of power series in two variables satisfying certain conditions. We provide a general argument to construct such a family (Subsection 2.6), which is applicable to other cases including the cases of type $A_{N-1}^{(t)}(t=1,2)$. We further show that the resulting functor $\mathcal{F}^{\prime}: \mathcal{T}_{2 n} \rightarrow \mathscr{C}_{B_{n}^{(1)}}^{0}$ induces a ring isomorphism from $K\left(\mathcal{T}_{2 n}\right)_{q=1}$ to $K\left(\mathscr{C}_{B_{n}^{(1)}}^{0}\right)$, which is bijective between the sets of classes of simple objects.

An immediate but surprising consequence of the main result is that for each $t=1,2$, there exists a ring isomorphism between $K\left(\mathscr{C}_{A_{2 n-1}^{(t)}}^{(}\right)$and $K\left(\mathscr{C}_{B_{n}^{(1)}}^{(1)}\right)$, which induces a bijection between the classes of simple modules. We believe that the correspondence here between the quantum affine algebra $U_{q}^{\prime}\left(B_{n}^{(1)}\right)$ and $U_{q}^{\prime}\left(A_{2 n-1}^{(2)}\right)$ is related to the fact that they are Langlands dual to each other; i.e., their Dynkin diagrams are obtained from each other by changing the directions of arrows. This speculation is supported heuristically by the studies on some small subcategories of $\mathscr{C}_{\mathfrak{g}}^{0}$. Recall that in $[12,15]$, certain tensor subcategories $\mathscr{C}_{Q}^{(t)}$ of $\mathscr{C}_{\mathfrak{g}}$, where $\mathfrak{g}=A_{2 n-1}^{(t)}$ or $D_{n+1}^{(t)}(t=1,2)$, are studied using the KLR-type quantum affine Schur-Weyl duality functors and it turns out that for each $t=1,2$, there is a ring isomorphism between the Grothendieck rings $K\left(R^{\mathfrak{g}_{0}}-\operatorname{gmod}\right)_{q=1}$ and $K\left(\mathscr{C}_{Q}^{(t)}\right)$, where $R^{\mathfrak{g}_{0}}$-gmod is the category of finite-dimensional graded modules over the symmetric quiver Hecke algebra of type $\mathfrak{g}_{0}=A_{2 n-1}$ or $D_{n+1}$. It induces also a bijection between the sets of classes of simple modules. In [19], the Langlands dual version $\mathscr{C}_{\mathscr{Q}}$ of the category $\mathscr{C}_{Q}$ is investigated. That is, there exist subcategories $\mathscr{C}_{\mathscr{Q}}$ of $\mathscr{C}_{\mathfrak{g}}$ where $\mathfrak{g}=B_{n}^{(1)}$ or $C_{n}^{(1)}$, and there is a ring isomorphism between the Grothendieck rings $K\left(R^{\mathfrak{g}_{0}} \text {-gmod }\right)_{q=1}$ and $K\left(\mathscr{C}_{\mathscr{Q}}\right)$, which induces a bijection between the sets of classes of simple modules. As a consequence, we have ring isomorphisms between the Grothendieck rings $K\left(\mathscr{C}_{Q}\right), K\left(\mathscr{C}_{\mathscr{Q}}\right)$, and $K\left(R^{\mathfrak{g}_{0}}-\operatorname{gmod}\right)_{q=1}$ which are bijective between the sets of classes of simple modules. Thus the main result of the present paper can be regarded as a global version of [19] in the case of type $A$ and type $B$ quantum affine algebras.

Recall that in [5] and [6], some interesting connections between the categories of finite-dimensional integrable representations over a quantum affine algebra and that of its Langlands dual are suggested and studied. Since the category $\mathscr{C}_{\mathfrak{g}}^{0}$ can be regarded as a skeleton of the category $\mathscr{C}_{\mathfrak{g}}$ of all the finite-dimensional integrable representations, the correspondence between the categories $\mathscr{C}_{A_{2 n-1}^{(2)}}^{0}$ and $\mathscr{C}_{B_{n}^{(1)}}^{0}$ in the present paper looks relevant to their works. It would be interesting to find a connection between the result in this paper and those in $[5,6]$. 
This paper is organized as follows. In the first section, we recall some necessary materials such as symmetric quiver Hecke algebras, quantum affine algebras, $R$-matrices and KLR-type quantum affine Schur-Weyl duality functors. In the second section, we present and study the KLR-type quantum affine Schur-Weyl duality functor $\mathcal{F}$ from $\mathcal{A}$ to $\mathscr{C}_{B_{n}^{(1)}}^{(0)}$. We show that $\mathcal{F}$ factors through the category $\mathcal{T}_{2 n}$ and the resulting functor induces an isomorphism between the Grothendieck rings, which is a bijection between the sets of classes of simple objects. In the last section, we record the images inside the category $\mathscr{C}_{B_{n}^{(1)}}^{0}$ of the fundamental representations in $\mathscr{C}_{A_{2 n-1}^{(t)}}^{0}(t=1,2)$ under the correspondence between the Grothendieck rings.

\section{Symmetric quiver Hecke algebras and quantum affine algebras}

1.1. Cartan datum and quantum groups. In this subsection, we recall the definition of quantum groups. Let $I$ be an index set. A Cartan datum is a sextuple $\left(A, P, \Pi, P^{\vee}, \Pi^{\vee},(\cdot, \cdot)\right)$ consisting of

(a) an integer-valued matrix $A=\left(a_{i j}\right)_{i, j \in I}$, called the symmetrizable generalized Cartan matrix, which satisfies

(i) $a_{i i}=2(i \in I)$,

(ii) $a_{i j} \leq 0(i \neq j)$,

(iii) $a_{i j}=0$ if $a_{j i}=0(i, j \in I)$,

(b) a free abelian group $P$, called the weight lattice,

(c) $\Pi=\left\{\alpha_{i} \in P \mid i \in I\right\}$, called the set of simple roots,

(d) $P^{\vee}:=\operatorname{Hom}(P, \mathbb{Z})$, called the co-weight lattice,

(e) $\Pi^{\vee}=\left\{h_{i} \mid i \in I\right\} \subset P^{\vee}$, called the set of simple coroots,

(f) a symmetric $\mathbb{Q}$-valued bilinear form $(\cdot, \cdot)$ on $P$,

satisfying the following properties:

(i) $\left\langle h_{i}, \alpha_{j}\right\rangle=a_{i j}$ for all $i, j \in I$,

(ii) $\Pi$ is linearly independent,

(iii) for each $i \in I$, there exists $\Lambda_{i} \in P$ such that $\left\langle h_{j}, \Lambda_{i}\right\rangle=\delta_{i j}$ for all $j \in I$,

(iv) $\left(\alpha_{i}, \alpha_{i}\right) \in \mathbb{Q}_{>0}$ for any $i \in I$,

(v) for any $\lambda \in P$ and $i \in I$, one has

$$
\left\langle h_{i}, \lambda\right\rangle=\frac{2\left(\alpha_{i}, \lambda\right)}{\left(\alpha_{i}, \alpha_{i}\right)} .
$$

We call $\Lambda_{i}$ the fundamental weights. The free abelian group $Q:=\bigoplus_{i \in I} \mathbb{Z} \alpha_{i}$ is called the root lattice. Set $\mathrm{Q}^{+}=\sum_{i \in I} \mathbb{Z}_{\geq 0} \alpha_{i} \subset \mathrm{Q}$ and $\mathrm{Q}^{-}=\sum_{i \in I} \mathbb{Z}_{\leq 0} \alpha_{i} \subset \mathrm{Q}$. For $\beta=\sum_{i \in I} m_{i} \alpha_{i} \in \mathrm{Q}$, we set $|\beta|=\sum_{i \in I}\left|m_{i}\right|$.

Let $d$ be the smallest positive integer such that $d \frac{\left(\alpha_{i}, \alpha_{i}\right)}{2} \in \mathbb{Z}$ for all $i \in I$. Let $q$ be an indeterminate. For each $i \in I$, set $q_{i}=q^{\frac{\left(\alpha_{i}, \alpha_{i}\right)}{2}} \in \mathbb{Q}\left(q^{1 / d}\right)$.

Definition 1.1.1. The quantum group $U_{q}(\mathfrak{g})$ associated with a Cartan datum $\left(A, P, \Pi, P^{\vee}, \Pi^{\vee},(\cdot, \cdot)\right)$ is the algebra over $\mathbb{Q}\left(q^{1 / d}\right)$ generated by $e_{i}, f_{i}(i \in I)$ and $q^{h}\left(h \in d^{-1} P^{\vee}\right)$ satisfying 
following relations:

$$
\begin{aligned}
& q^{0}=1, \quad q^{h} q^{h^{\prime}}=q^{h+h^{\prime}} \text { for } h, h^{\prime} \in d^{-1} P^{\vee}, \\
& q^{h} e_{i} q^{-h}=q^{\left\langle h, \alpha_{i}\right\rangle} e_{i}, \quad q^{h} f_{i} q^{-h}=q^{-\left\langle h, \alpha_{i}\right\rangle} f_{i} \quad \text { for } h \in d^{-1} P^{\vee}, i \in I, \\
& e_{i} f_{j}-f_{j} e_{i}=\delta_{i j} \frac{K_{i}-K_{i}^{-1}}{q_{i}-q_{i}^{-1}} \quad \text { where } K_{i}=q^{\frac{\left(\alpha_{i}, \alpha_{i}\right)}{2} h_{i}}, \\
& \sum_{r=0}^{1-a_{i j}}(-1)^{r} e_{i}^{\left(1-a_{i j}-r\right)} e_{j} e_{i}^{(r)}=\sum_{r=0}^{1-a_{i j}}(-1)^{r} f_{i}^{\left(1-a_{i j}-r\right)} f_{j} f_{i}^{(r)}=0 \quad \text { if } i \neq j .
\end{aligned}
$$

Here, we set $[n]_{i}=\frac{q_{i}^{n}-q_{i}^{-n}}{q_{i}-q_{i}^{-1}}, \quad[n]_{i} !=\prod_{k=1}^{n}[k]_{i}, e_{i}^{(m)}=\frac{e_{i}^{n}}{[n]_{i} !}$ and $f_{i}^{(n)}=\frac{f_{i}^{n}}{[n]_{i} !}$ for all $n \in \mathbb{Z}_{\geq 0}, i \in I$.

1.2. Quiver Hecke algebras. We recall the definition of quiver Hecke algebras associated with a given Cartan datum $\left(A, P, \Pi, P^{\vee}, \Pi^{\vee},(\cdot, \cdot)\right)$ such that $\left(\alpha_{i}, \alpha_{i}\right) \in 2 \mathbb{Z}$ for all $i \in I$.

Let $\mathbf{k}$ be a commutative ring. Let us take a family of polynomials $\left(Q_{i, j}\right)_{i, j \in I}$ in $\mathbf{k}[u, v]$ which satisfies

$$
\begin{aligned}
& \text { (a) } Q_{i, j}(u, v)=Q_{j, i}(v, u) \text { for any } i, j \in I, \\
& \text { (b) } Q_{i, j}(u, v)=0 \text { for } i=j \in I, \\
& \text { (c) for } i \neq j \text {, we have } \\
& \qquad Q_{i, j}(u, v)=\sum_{\substack{(p, q) \in \mathbb{Z}_{\geq 0}^{2} \\
\left(\alpha_{i}, \alpha_{i}\right) p+\left(\alpha_{j}, \alpha_{j}\right) q=-2\left(\alpha_{i}, \alpha_{j}\right)}} t_{i, j ; p, q} u^{p} v^{q} \\
& \text { with } t_{i, j ; p, q} \in \mathbf{k}, t_{i, j ; p, q}=t_{j, i ; q, p} \text { and } t_{i, j:-a_{i j}, 0} \in \mathbf{k}^{\times} .
\end{aligned}
$$

We denote by $\mathfrak{S}_{n}=\left\langle s_{1}, \ldots, s_{n-1}\right\rangle$ the symmetric group on $n$ letters, where $s_{i}:=$ $(i, i+1)$ is the transposition of $i$ and $i+1$. Then $\mathfrak{S}_{n}$ acts on $I^{n}$ by place permutations.

For $n \in \mathbb{Z}_{\geq 0}$ and $\beta \in \mathrm{Q}^{+}$such that $|\beta|=n$, we set

$$
I^{\beta}=\left\{\nu=\left(\nu_{1}, \ldots, \nu_{n}\right) \in I^{n} ; \alpha_{\nu_{1}}+\cdots+\alpha_{\nu_{n}}=\beta\right\} .
$$

Definition 1.2.1. For $\beta \in \mathrm{Q}^{+}$with $|\beta|=n$, the quiver Hecke algebra $R(\beta)$ at $\beta$ associated with a Cartan datum $\left(A, P, \Pi, P^{\vee}, \Pi^{\vee},(\cdot, \cdot)\right)$ and a matrix $\left(Q_{i, j}\right)_{i, j \in I}$ is the k-algebra generated by the elements $\{e(\nu)\}_{\nu \in I^{\beta}},\left\{x_{k}\right\}_{1 \leq k \leq n},\left\{\tau_{m}\right\}_{1 \leq m \leq n-1}$ satisfying the following defining relations:

$$
\begin{aligned}
& e(\nu) e\left(\nu^{\prime}\right)=\delta_{\nu, \nu^{\prime}} e(\nu), \quad \sum_{\nu \in I^{\beta}} e(\nu)=1, \\
& x_{k} x_{m}=x_{m} x_{k}, \quad x_{k} e(\nu)=e(\nu) x_{k}, \\
& \tau_{m} e(\nu)=e\left(s_{m}(\nu)\right) \tau_{m}, \quad \tau_{k} \tau_{m}=\tau_{m} \tau_{k} \quad \text { if }|k-m|>1, \\
& \tau_{k}^{2} e(\nu)=Q_{\nu_{k}, \nu_{k+1}}\left(x_{k}, x_{k+1}\right) e(\nu),
\end{aligned}
$$




$$
\begin{aligned}
& \left(\tau_{k} x_{m}-x_{s_{k}(m)} \tau_{k}\right) e(\nu)= \begin{cases}-e(\nu) & \text { if } m=k, \nu_{k}=\nu_{k+1}, \\
e(\nu) & \text { if } m=k+1, \nu_{k}=\nu_{k+1}, \\
0 & \text { otherwise, }\end{cases} \\
& \left(\tau_{k+1} \tau_{k} \tau_{k+1}-\tau_{k} \tau_{k+1} \tau_{k}\right) e(\nu) \\
& = \begin{cases}\frac{Q_{\nu_{k}, \nu_{k+1}}\left(x_{k}, x_{k+1}\right)-Q_{\nu_{k}, \nu_{k+1}}\left(x_{k+2}, x_{k+1}\right)}{x_{k}-x_{k+2}} e(\nu) & \text { if } \nu_{k}=\nu_{k+2}, \\
0 & \text { otherwise. }\end{cases}
\end{aligned}
$$

Note that $R(\beta)$ is a $\mathbb{Z}$-graded algebra provided with

$$
\operatorname{deg} e(\nu)=0, \quad \operatorname{deg} x_{k} e(\nu)=\left(\alpha_{\nu_{k}}, \alpha_{\nu_{k}}\right), \quad \operatorname{deg} \tau_{l} e(\nu)=-\left(\alpha_{\nu_{l}}, \alpha_{\nu_{l+1}}\right) .
$$

Let us denote by $\operatorname{Mod}(R(\beta))$ the category of $R(\beta)$-modules and by $\operatorname{Mod}_{\mathrm{gr}}(R(\beta))$ the category of graded $R(\beta)$-modules. The category of graded $R(\beta)$-modules which are finite-dimensional over $\mathbf{k}$ is denoted by $R(\beta)$-gmod.

In this paper, an $R(\beta)$-module means a graded $R(\beta)$-module, unless stated otherwise.

For a graded $R(\beta)$-module $M=\bigoplus_{k \in \mathbb{Z}} M_{k}$, we define $q M=\bigoplus_{k \in \mathbb{Z}}(q M)_{k}$, where

$$
(q M)_{k}=M_{k-1}(k \in \mathbb{Z}) .
$$

We call $q$ the grading shift functor on the category of graded $R(\beta)$-modules.

For $\beta, \gamma \in \mathbf{Q}^{+}$with $|\beta|=m,|\gamma|=n$, set

$$
e(\beta, \gamma)=\sum_{\substack{\nu \in I^{m+n},\left(\nu_{1}, \ldots, \nu_{m}\right) \in I^{\beta},\left(\nu_{m+1}, \ldots, \nu_{m+n}\right) \in I^{\gamma}}} e(\nu) .
$$

Then $e(\beta, \gamma)$ is an idempotent element of $R(\beta+\gamma)$. Let

$$
R(\beta) \otimes R(\gamma) \rightarrow e(\beta, \gamma) R(\beta+\gamma) e(\beta, \gamma)
$$

be the $\mathbf{k}$-algebra homomorphism given by

$$
\begin{aligned}
& e(\mu) \otimes e(\nu) \mapsto e(\mu * \nu) \quad\left(\mu \in I^{\beta}\right), \\
& x_{k} \otimes 1 \mapsto x_{k} e(\beta, \gamma) \quad(1 \leq k \leq m), \\
& 1 \otimes x_{k} \mapsto x_{m+k} e(\beta, \gamma) \quad(1 \leq k \leq n), \\
& \tau_{k} \otimes 1 \mapsto \tau_{k} e(\beta, \gamma) \quad(1 \leq k<m), \\
& 1 \otimes \tau_{k} \mapsto \tau_{m+k} e(\beta, \gamma) \quad(1 \leq k<n),
\end{aligned}
$$

where $\mu * \nu$ is the concatenation of $\mu$ and $\nu$; i.e., $\mu * \nu=\left(\mu_{1}, \ldots, \mu_{m}, \nu_{1}, \ldots, \nu_{n}\right)$.

For an $R(\beta)$-module $M$ and an $R(\gamma)$-module $N$, we define the convolution product $M \circ N$ by

$$
M \circ N:=R(\beta+\gamma) e(\beta, \gamma) \underset{R(\beta) \otimes R(\gamma)}{\otimes}(M \otimes N) .
$$


1.3. R-matrices for quiver Hecke algebra. For $|\beta|=n$ and $1 \leq a<n$, we define $\varphi_{a} \in R(\beta)$ by

$$
\varphi_{a} e(\nu)= \begin{cases}\left(\tau_{a} x_{a}-x_{a} \tau_{a}\right) e(\nu) & \text { if } \nu_{a}=\nu_{a+1}, \\ \tau_{a} e(\nu) & \text { otherwise. }\end{cases}
$$

They are called the intertwiners. Since $\left\{\varphi_{k}\right\}_{1 \leq k \leq n-1}$ satisfies the braid relation, we have a well-defined element $\varphi_{w} \in R(\beta)$ for each $w \in \mathfrak{S}_{n}$.

For $m, n \in \mathbb{Z}_{\geq 0}$, we set

$$
\mathfrak{S}_{m, n}:=\left\{w \in \mathfrak{S}_{m+n} ; w(i)<w(i+1) \text { for any } i \neq m\right\} .
$$

For example,

$$
w[m, n](k)= \begin{cases}k+n & \text { if } 1 \leq k \leq m, \\ k-m & \text { if } m<k \leq m+n\end{cases}
$$

is an element in $\mathfrak{S}_{m, n}$.

Let $\beta, \gamma \in \mathrm{Q}^{+}$with $|\beta|=m,|\gamma|=n$ and let $M$ be an $R(\beta)$-module and let $N$ be an $R(\gamma)$-module. Then the map

$$
M \otimes N \rightarrow q^{(\beta, \gamma)-2(\beta, \gamma)_{\mathrm{n}}} N \circ M
$$

given by

$$
u \otimes v \longmapsto \varphi_{w[n, m]}(v \otimes u)
$$

is an $R(\beta, \gamma)$-module homomorphism by [11, Lemma 1.3.1], and it extends to an $R(\beta+$ $\gamma)$-module homomorphism

$$
\mathrm{R}_{M, N}: M \circ N \longrightarrow q^{(\beta, \gamma)-2(\beta, \gamma)_{\mathrm{n}}} N \circ M
$$

where the symmetric bilinear form $(\bullet, \bullet)_{\mathrm{n}}$ on $\mathrm{Q}$ is given by $\left(\alpha_{i}, \alpha_{j}\right)_{\mathrm{n}}=\delta_{i j}$.

Definition 1.3.1. A quiver Hecke algebra $R(\beta)$ is symmetric if $Q_{i, j}(u, v)$ is a polynomial in $\mathbf{k}[u-v]$ for all $i, j \in I$.

From now on, we assume that quiver Hecke algebras are symmetric. Then, the generalized Cartan matrix $A=\left(a_{i j}\right)_{i, j \in I}$ is symmetric. We assume then $\left(\alpha_{i}, \alpha_{j}\right)=a_{i, j}$. Let $z$ be an indeterminate which is homogeneous of degree 2 , and let $\psi_{z}$ be the algebra homomorphism

$$
\psi_{z}: R(\beta) \rightarrow \mathbf{k}[z] \otimes R(\beta)
$$

given by

$$
\psi_{z}\left(x_{k}\right)=x_{k}+z, \quad \psi_{z}\left(\tau_{k}\right)=\tau_{k}, \quad \psi_{z}(e(\nu))=e(\nu) .
$$

For an $R(\beta)$-module $M$, we denote by $M_{z}$ the $(\mathbf{k}[z] \otimes R(\beta))$-module $\mathbf{k}[z] \otimes M$ with the action of $R(\beta)$ twisted by $\psi_{z}$. Namely,

$$
\begin{aligned}
& e(\nu)(a \otimes u)=a \otimes e(\nu) u, \\
& x_{k}(a \otimes u)=(z a) \otimes u+a \otimes\left(x_{k} u\right), \\
& \tau_{k}(a \otimes u)=a \otimes\left(\tau_{k} u\right)
\end{aligned}
$$


for $\nu \in I^{\beta}, a \in \mathbf{k}[z]$ and $u \in M$. For $u \in M$, we sometimes denote by $u_{z}$ the corresponding element $1 \otimes u$ of the $R(\beta)$-module $M_{z}$.

For a non-zero $R(\beta)$-module $M$ and a non-zero $R(\gamma)$-module $N$,

let $s$ be the order of zero of $R_{M_{z}, N_{z^{\prime}}}: M_{z} \circ N_{z^{\prime}} \longrightarrow q^{(\beta, \gamma)-2(\beta, \gamma)_{\mathrm{n}}} N_{z^{\prime}} \circ M_{z}$,

i.e., the largest non-negative integer such that the image of $R_{M_{z}, N_{z^{\prime}}}$ is contained in $\left(z^{\prime}-z\right)^{s} q^{(\beta, \gamma)-2(\beta, \gamma)_{\mathrm{n}}} N_{z^{\prime}} \circ M_{z}$.

Note that [11, Proposition 1.4.4 (iii)] shows that such an $s$ exists.

Definition 1.3.2. For a non-zero $R(\beta)$-module $M$ and a non-zero $R(\gamma)$-module $N$, we set

$$
\Lambda(M, N):=-(\beta, \gamma)+2(\beta, \gamma)_{\mathrm{n}}-2 s, \quad R_{M_{z}, N_{z^{\prime}}}^{\mathrm{ren}}:=\left(z^{\prime}-z\right)^{-s} R_{M_{z}, N_{z^{\prime}}}
$$

and define

$$
\mathbf{r}_{M, N}: M \circ N \rightarrow q^{-\Lambda(M, N)} N \circ M
$$

by

$$
\mathbf{r}_{M, N}=\left.\left(R_{M_{z}, N_{z^{\prime}}}^{\mathrm{ren}}\right)\right|_{z=z^{\prime}=0}
$$

By [11, Proposition 1.4.4 (ii)], the morphism $\mathbf{r}_{M, N}$ does not vanish if $M$ and $N$ are non-zero.

Lemma 1.3.3 ([14]). Let $M$ and $N$ be simple $R$-modules. Then we have

(i) $\Lambda(M, N)+\Lambda(N, M) \in 2 \mathbb{Z}_{\geq 0}$.

(ii) If $\Lambda(M, N)+\Lambda(N, M)=2 m$ for some $m \in \mathbb{Z}_{\geq 0}$, then

$$
R_{M_{z}, N}^{\mathrm{ren}} \circ R_{N, M_{z}}^{\mathrm{ren}}=z^{m} \mathrm{id}_{N \circ M_{z}} \quad \text { and } \quad R_{N, M_{z}}^{\mathrm{ren}} \circ R_{M_{z}, N}^{\mathrm{ren}}=z^{m} \operatorname{id}_{M_{z} \circ N}
$$

up to constant multiples.

For simple modules $M$ and $N$, we set

$$
\mathfrak{D}(M, N)=\frac{1}{2}(\Lambda(M, N)+\Lambda(N, M)) \in \mathbb{Z}_{\geq 0} .
$$

A simple module $M \in R$-gmod is called real if $M \circ M$ is simple.

Lemma 1.3.4 ([13]). Let $M$ and $N$ be simple modules in $R$-gmod, and assume that one of them is real. Then

(i) $M \circ N$ and $N \circ M$ have simple socles and simple heads.

(ii) Moreover, $\operatorname{Im}\left(\mathbf{r}_{M, N}\right)$ is equal to the head of $M \circ N$ and the socle of $N \circ M$. Similarly, $\operatorname{Im}\left(\mathbf{r}_{N, M}\right)$ is equal to the head of $N \circ M$ and the socle of $M \circ N$ (up to grading shifts).

For $M, N \in R$-gmod, we denote by $M \nabla N$ and $M \Delta N$ the head and the socle of $M \circ N$, respectively.

The number $\mathfrak{D}(M, N)$ measures the degree of complexity of $M \circ N$ as seen in the following lemma.

Lemma 1.3.5 ([16, Proposition 4.14]). Let $M$ and $N$ be simple modules in $R$-gmod, and assume that one of them is real. 
(i) $M \circ N$ is simple if and only if $\mathrm{D}(M, N)=0$.

(ii) If $\triangleright(M, N)=1$, then $M \circ N$ has length 2 , and there exists an exact sequence

$$
0 \rightarrow M \Delta N \rightarrow M \circ N \rightarrow M \nabla N \rightarrow 0 \text {. }
$$

1.4. Quantum affine algebras. In this subsection, we briefly review the representation theory of finite-dimensional integrable modules over quantum affine algebras following $[1,18]$. When concerned with quantum affine algebras, we take the algebraic closure of $\mathbb{C}(q)$ in $\cup_{m>0} \mathbb{C}\left(\left(q^{1 / m}\right)\right)$ as the base field $\mathbf{k}$.

Let $I$ be an index set and let $A=\left(a_{i j}\right)_{i, j \in I}$ be a generalized Cartan matrix of affine type. We choose $0 \in I$ as the leftmost vertices in the tables in [10, pages 54,55$]$ except $A_{2 n}^{(2)}$-case in which case we take the longest simple root as $\alpha_{0}$. Set $I_{0}=I \backslash\{0\}$.

The weight lattice $P$ is given by

$$
P=\left(\bigoplus_{i \in I} \mathbb{Z} \Lambda_{i}\right) \oplus \mathbb{Z} \delta
$$

and the simple roots are given by

$$
\alpha_{i}=\sum_{j \in I} a_{j i} \Lambda_{j} \text { for } i \in I_{0}, \quad \text { and } \quad \alpha_{0}=\sum_{j \in I} a_{j 0} \Lambda_{j}+\delta .
$$

The weight $\delta$ is called the imaginary root. There exist $d_{i} \in \mathbb{Z}_{>0}$ such that

$$
\delta=\sum_{i \in I} d_{i} \alpha_{i}
$$

Note that $d_{i}=1$ for $i=0$. The simple coroots $h_{i} \in P^{\vee}$ are given by

$$
\left\langle h_{i}, \Lambda_{j}\right\rangle=\delta_{i j}, \quad\left\langle h_{i}, \delta\right\rangle=0 .
$$

Let $c=\sum_{i \in I} c_{i} h_{i}$ be a unique element such that $c_{i} \in \mathbb{Z}_{>0}$ and

$$
\mathbb{Z} c=\left\{h \in \bigoplus_{i \in I} \mathbb{Z} h_{i} ;\left\langle h, \alpha_{i}\right\rangle=0 \text { for any } i \in I\right\} .
$$

We normalize the $\mathbb{Q}$-valued symmetric bilinear form $(\bullet, \bullet)$ on $P$ by

$$
(\delta, \lambda)=\langle c, \lambda\rangle \text { for any } \lambda \in P .
$$

Let us denote by $U_{q}(\mathfrak{g})$ the quantum group associated with the affine Cartan datum $\left(A, P, \Pi, P^{\vee}, \Pi^{\vee},(\cdot, \cdot)\right)$. We denote by $U_{q}^{\prime}(\mathfrak{g})$ the subalgebra of $U_{q}(\mathfrak{g})$ generated by $e_{i}, f_{i}, K_{i}^{ \pm 1}$ for $i \in I$. We call $U_{q}^{\prime}(\mathfrak{g})$ the quantum affine algebra associated with the generalized Cartan matrix $A$. Let us denote by $\operatorname{Mod}\left(U_{q}^{\prime}(\mathfrak{g})\right)$ the category of left modules of $U_{q}^{\prime}(\mathfrak{g})$.

The algebra $U_{q}^{\prime}(\mathfrak{g})$ has a Hopf algebra structure with the following coproduct $\Delta$, counit $\varepsilon$, and antipode $S$ :

$$
\begin{aligned}
& \Delta\left(K_{i}\right)=K_{i} \otimes K_{i}, \quad \Delta\left(e_{i}\right)=e_{i} \otimes K_{i}^{-1}+1 \otimes e_{i}, \quad \Delta\left(f_{i}\right)=f_{i} \otimes 1+K_{i} \otimes f_{i}, \\
& \varepsilon\left(K_{i}\right)=1, \quad \varepsilon\left(e_{i}\right)=\varepsilon\left(f_{i}\right)=0, \\
& S\left(K_{i}\right)=K_{i}^{-1}, \quad S\left(e_{i}\right)=-e_{i} K_{i}, \quad S\left(f_{i}\right)=-K_{i}^{-1} f_{i} .
\end{aligned}
$$

Set

$$
P_{\mathrm{cl}}=P / \mathbb{Z} \delta
$$


and call it the classical weight lattice. Let $\mathrm{cl}: P \rightarrow P_{\mathrm{cl}}$ denote the projection. Then $P_{\mathrm{cl}}=\bigoplus_{i \in I} \mathbb{Z} \mathrm{cl}\left(\Lambda_{i}\right)$. Set $P_{\mathrm{cl}}^{0}=\left\{\lambda \in P_{\mathrm{cl}} ;\langle c, \lambda\rangle=0\right\} \subset P_{\mathrm{cl}}$.

Let us denote by $W$ the Weyl group, i.e., the subgroup of $\operatorname{Aut}(P)$ generated by the simple reflections $s_{i}(i \in I)$, where $s_{i}(\lambda)=\lambda-\left\langle h_{i}, \lambda\right\rangle(\lambda \in P)$. Then $W$ acts also on $P_{\mathrm{cl}}$ and $P_{\mathrm{cl}}^{0}$.

A $U_{q}^{\prime}(\mathfrak{g})$-module $M$ is called an integrable module if

(a) $M$ has a weight space decomposition

$$
M=\bigoplus_{\lambda \in P_{\mathrm{cl}}} M_{\lambda}
$$

where $M_{\lambda}=\left\{u \in M ; K_{i} u=q_{i}^{\left\langle h_{i}, \lambda\right\rangle} u\right.$ for all $\left.i \in I\right\}$,

(b) the actions of $e_{i}$ and $f_{i}$ on $M$ are locally nilpotent for any $i \in I$.

Let us denote by $\mathscr{C}_{\mathfrak{g}}$ the abelian tensor category of finite-dimensional integrable $U_{q}^{\prime}(\mathfrak{g})$-modules.

If $M$ is a simple module in $\mathscr{C}_{\mathfrak{g}}$, then there exists a non-zero vector $u \in M$ of weight $\lambda \in P_{\mathrm{cl}}^{0}$ such that $\lambda$ is dominant (i.e., $\left\langle h_{i}, \lambda\right\rangle \geq 0$ for any $i \in I_{0}$ ) and all the weights of $M$ lie in $\lambda-\sum_{i \in I_{0}} \mathbb{Z}_{\geq 0} \operatorname{cl}\left(\alpha_{i}\right)$. Such a $\lambda$ is unique and called the dominant extremal weight of $M$. Moreover, we have $\operatorname{dim} M_{\lambda}=1$, and a non-zero vector of $M_{\lambda}$ is called a dominant extremal vector of $M$.

Let $M$ be an integrable $U_{q}^{\prime}(\mathfrak{g})$-module. Then the affinization $M_{\text {aff }}$ of $M$ is the space $M_{\text {aff }}=\mathbf{k}\left[z, z^{-1}\right] \otimes M$ with a $\mathbf{k}\left[z, z^{-1}\right] \otimes U_{q}^{\prime}(\mathfrak{g})$-action given by

$$
e_{i}\left(u_{z}\right)=z^{\delta_{i, 0}}\left(e_{i} u\right)_{z}, \quad f_{i}\left(u_{z}\right)=z^{-\delta_{i, 0}}\left(f_{i} u\right)_{z}, \quad K_{i}\left(u_{z}\right)=\left(K_{i} u\right)_{z},
$$

where $u_{z}$ denotes the element $\mathbf{1} \otimes u \in M_{\text {aff }}$ for $u \in M$. We denote the action of $z$ on $M_{\text {aff }}$ by $z_{M}$. We sometimes write $\left(M_{z}, z\right)$ for $\left(M_{\mathrm{aff}}, z_{M}\right)$.

For $x \in \mathbf{k}^{\times}$, we define

$$
M_{x}:=M_{\mathrm{aff}} /\left(z_{M}-x\right) M_{\mathrm{aff}} .
$$

We sometimes call $x$ the spectral parameter.

Set $\varpi_{i}=\operatorname{gcd}\left(c_{0}, c_{i}\right)^{-1} \operatorname{cl}\left(c_{0} \Lambda_{i}-c_{i} \Lambda_{0}\right) \in P_{\mathrm{cl}}$ for $i \in I_{0}$. Then $\left\{\varpi_{i}\right\}_{i \in I_{0}}$ forms a basis of $P_{\mathrm{cl}}^{0}$. We call $\varpi_{i}$ a (level 0$)$ fundamental weight.

Then for any $i \in I_{0}$, there exists a non-zero $U_{q}^{\prime}(\mathfrak{g})$-module $M$ in $\mathscr{C}_{\mathfrak{g}}$ satisfying the following properties: we can take $u_{\lambda} \in M_{\lambda}$ for each $\lambda \in W \varpi_{i} \subset P_{\mathrm{cl}}$ such that

(a) if $j \in I$ and $\lambda \in W \varpi_{i}$ satisfy $\left\langle h_{j}, \lambda\right\rangle \geq 0$, then $e_{j} u_{\lambda}=0$ and $f_{j}^{\left(\left\langle h_{j}, \lambda\right\rangle\right)} u_{\lambda}=u_{s_{j} \lambda}$

(b) if $j \in I$ and $\lambda \in W \varpi_{i}$ satisfy $\left\langle h_{j}, \lambda\right\rangle \leq 0$, then $f_{j} u_{\lambda}=0$ and $e_{j}^{\left(-\left\langle h_{j}, \lambda\right\rangle\right)} u_{\lambda}=u_{s_{j} \lambda}$,

(c) $M$ is generated by $u_{\varpi_{i}}$.

Then $M$ is a simple module with a dominant extremal weight $\varpi_{i}$ and it is unique up to an isomorphism ([18]). We call $M$ the fundamental representation with dominant extremal weight $\varpi_{i}$, and denote it by $V\left(\varpi_{i}\right)$. 
With a slight abuse of terminology, we also call $V\left(\varpi_{i}\right)_{x}\left(x \in \mathbf{k}^{\times}\right)$a fundamental representation.

If a $U_{q}^{\prime}(\mathfrak{g})$-module $M \in \mathscr{C}_{\mathfrak{g}}$ has a bar involution, a crystal basis with simple crystal graph and a lower global basis, then we say that $M$ is a good module. For the precise definition, see $[18, \S 8]$. Every good module is a simple $U_{q}^{\prime}(\mathfrak{g})$-module and a tensor product of a good module is again a good module. For example, the fundamental representation $V\left(\varpi_{i}\right)$ is a good $U_{q}^{\prime}(\mathfrak{g})$-module.

For a module $M$ in $\mathscr{C}_{\mathfrak{g}}$, let us denote the right and the left dual of $M$ by ${ }^{*} M$ and $M^{*}$, respectively. That is, we have isomorphisms

$$
\begin{aligned}
& \operatorname{Hom}_{U_{q}^{\prime}(\mathfrak{g})}(M \otimes X, Y) \simeq \operatorname{Hom}_{U_{q}^{\prime}(\mathfrak{g})}\left(X,{ }^{*} M \otimes Y\right), \\
& \operatorname{Hom}_{U_{q}^{\prime}(\mathfrak{g})}\left(X \otimes{ }^{*} M, Y\right) \simeq \operatorname{Hom}_{U_{q}^{\prime}(\mathfrak{g})}(X, Y \otimes M), \\
& \operatorname{Hom}_{U_{q}^{\prime}(\mathfrak{g})}\left(M^{*} \otimes X, Y\right) \simeq \operatorname{Hom}_{U_{q}^{\prime}(\mathfrak{g})}(X, M \otimes Y), \\
& \operatorname{Hom}_{U_{q}^{\prime}(\mathfrak{g})}(X \otimes M, Y) \simeq \operatorname{Hom}_{U_{q}^{\prime}(\mathfrak{g})}\left(X, Y \otimes M^{*}\right)
\end{aligned}
$$

which are functorial in $U_{q}^{\prime}(\mathfrak{g})$-modules $X, Y$. Then we have ${ }^{*}\left(M_{x}\right)=\left({ }^{*} M\right)_{x}$ and $\left(M_{x}\right)^{*}=\left(M^{*}\right)_{x}$ for any $x \in \mathbf{k}^{\times}$. The dual of fundamental representations are as follows:

$$
{ }^{*} V\left(\varpi_{i}\right) \simeq V\left(\varpi_{i^{*}}\right)_{p^{*}}, \quad\left(V\left(\varpi_{i}\right)\right)^{*} \simeq V\left(\varpi_{i^{*}}\right)_{p^{*-1}}
$$

for $i \in I_{0}$. Here, $p^{*}=(-1)^{\left\langle\rho^{\vee}, \delta\right\rangle} q^{\langle c, \rho\rangle}$, and $\rho$ (respectively, $\rho^{\vee}$ ) denotes an element in $P$ (respectively, $P^{\vee}$ ) such that $\left\langle h_{i}, \rho\right\rangle=1$ (respectively $\left\langle\rho^{\vee}, \alpha_{i}\right\rangle=1$ ) for every $i \in I$. The map $i \mapsto i^{*}$ is the involution on $I_{0}$ determined by $\alpha_{i^{*}}=-w_{0} \alpha_{i}$, where $w_{0}$ denotes the longest element of $W_{0}=\left\langle s_{i} \mid i \in I_{0}\right\rangle \subset W$.

1.5. R-matrices for quantum affine algebras. We recall the notion of $R$-matrices for quantum affine algebras, For details see $[18, \S 8]$. Let us choose the following universal $R$-matrix. Let us take a basis $\left\{P_{\nu}\right\}_{\nu}$ of $U_{q}^{+}(\mathfrak{g})$ and a basis $\left\{Q_{\nu}\right\}_{\nu}$ of $U_{q}^{-}(\mathfrak{g})$ dual to each other with respect to a suitable coupling between $U_{q}^{+}(\mathfrak{g})$ and $U_{q}^{-}(\mathfrak{g})$. Then for $U_{q}^{\prime}(\mathfrak{g})$-modules $M$ and $N$ define

$$
R_{M N}^{\mathrm{univ}}(u \otimes v)=q^{(\mathrm{wt}(u), \mathrm{wt}(v))} \sum_{\nu} P_{\nu} v \otimes Q_{\nu} u
$$

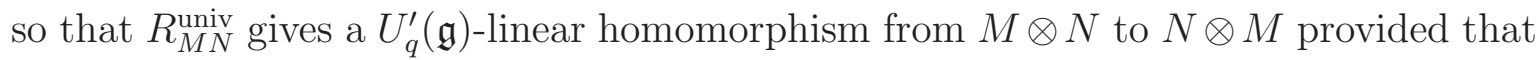
the infinite sum has a meaning.

Let $M$ and $N$ be $U_{q}^{\prime}(\mathfrak{g})$-modules in $\mathscr{C}_{\mathfrak{g}}$, and let $z_{1}$ and $z_{2}$ be indeterminates. Then $R_{M_{z_{1}}, N_{z_{2}}}^{\text {univ }}$ converges in the $\left(z_{2} / z_{1}\right)$-adic topology. Hence we obtain a morphism of $\mathbf{k}\left[\left[z_{2} / z_{1}\right]\right] \otimes_{\mathbf{k}\left[z_{2} / z_{1}\right]} \mathbf{k}\left[z_{1}^{ \pm 1}, z_{2}^{ \pm 1}\right] \otimes U_{q}^{\prime}(\mathfrak{g})$-modules

$$
R_{M_{z_{1}}, N_{z_{2}}}^{\text {univ }}: \mathbf{k}\left[\left[z_{2} / z_{1}\right]\right] \underset{\mathbf{k}\left[z_{2} / z_{1}\right]}{\otimes}\left(M_{z_{1}} \otimes N_{z_{2}}\right) \rightarrow \mathbf{k}\left[\left[z_{2} / z_{1}\right]\right] \underset{\mathbf{k}\left[z_{2} / z_{1}\right]}{\otimes}\left(N_{z_{2}} \otimes M_{z_{1}}\right) .
$$

If there exist $a \in \mathbf{k}\left(\left(z_{2} / z_{1}\right)\right)$ and a $\mathbf{k}\left[z_{1}^{ \pm 1}, z_{2}^{ \pm 1}\right] \otimes U_{q}^{\prime}(\mathfrak{g})$-linear homomorphism

$$
R_{M_{z_{1}}, N_{z_{2}}}^{\mathrm{ren}}: M_{z_{1}} \otimes N_{z_{2}} \rightarrow N_{z_{2}} \otimes M_{z_{1}}
$$


such that $R_{M_{z_{1}}, N_{z_{2}}}^{\text {univ }}=a R_{M_{z_{1}}, N_{z_{2}}}^{\mathrm{ren}}$, then we say that $R_{M_{z_{1}, N_{z_{2}}}}^{\text {univ }}$ is rationally renormalizable.

Now assume further that $M$ and $N$ are non-zero. Then, we can choose $R_{M_{z_{1}}, N_{z_{2}}}^{\text {son }}$ that, for any $c_{1}, c_{2} \in \mathbf{k}^{\times}$, the specialization of $R_{M_{z_{1}}, N_{z_{2}}}^{\mathrm{ren}}$ at $z_{1}=c_{1}, z_{2}=c_{2}$

$$
\left.R_{M_{z_{1}}, N_{z_{2}}}^{\mathrm{ren}}\right|_{z_{1}=c_{1}, z_{2}=c_{2}}: M_{c_{1}} \otimes N_{c_{2}} \rightarrow N_{c_{2}} \otimes M_{c_{1}}
$$

does not vanish. Such an $R^{\text {ren }}$ is unique up to a multiple of $\mathbf{k}\left[\left(z_{2} / z_{1}\right)^{ \pm 1}\right]^{\times}=\bigsqcup_{n \in \mathbb{Z}} \mathbf{k}^{\times}\left(z_{2} / z_{1}\right)^{n}$, and it is called a renormalized $R$-matrix. We write

$$
\mathbf{r}_{M, N}:=\left.R^{\text {ren }}\right|_{z_{1}=z_{2}=1}: M \otimes N \rightarrow N \otimes M,
$$

and call it the $R$-matrix between $M$ and $N$. The $R$-matrix $\mathbf{r}_{M, N}$ is well defined up to a constant multiple when $R_{M_{z_{1}}, N_{z_{2}}}^{\text {univ }}$ is rationally renormalizable. By the definition, $\mathbf{r}_{M, N}$ never vanishes.

Now assume that $M_{1}$ and $M_{2}$ are simple $U_{q}^{\prime}(\mathfrak{g})$-modules in $\mathscr{C}_{\mathfrak{g}}$. Then, the universal $R$ matrix $R_{\left(M_{1}\right)_{z_{1}},\left(M_{2}\right)_{z_{2}}}^{\text {univ }}$ is rationally renormalizable. More precisely, we have the following. Let $u_{1}$ and $u_{2}$ be dominant extremal weight vectors of $M_{1}$ and $M_{2}$, respectively. Then there exists $a_{M_{1} . M_{2}}\left(z_{2} / z_{1}\right) \in \mathbf{k}\left[\left[z_{2} / z_{1}\right]\right]^{\times}$such that

$$
R_{\left(M_{1}\right)_{z_{1}},\left(M_{2}\right)_{z_{2}}}^{\text {univ }}\left(\left(u_{1}\right)_{z_{1}} \otimes\left(u_{2}\right)_{z_{2}}\right)=a_{M_{1} . M_{2}}\left(z_{2} / z_{1}\right)\left(\left(u_{2}\right)_{z_{2}} \otimes\left(u_{1}\right)_{z_{1}}\right) .
$$

Then $R_{\left(M_{1}\right)_{z_{1}},\left(M_{2}\right)_{z_{2}}}^{\text {norm }}:=a_{M_{1} . M_{2}}\left(z_{2} / z_{1}\right)^{-1} R_{\left(M_{1}\right)_{z_{1}},\left(M_{2}\right)_{z_{2}}}^{\text {univ }}$ is a unique $\mathbf{k}\left(z_{1}, z_{2}\right) \otimes U_{q}^{\prime}(\mathfrak{g})$-module homomorphism

$$
\begin{aligned}
R_{\left(M_{1}\right)_{z_{1}},\left(M_{2}\right)_{z_{2}}}^{\mathrm{norm}}: \mathbf{k}\left(z_{1}, z_{2}\right) \otimes_{\mathbf{k}\left[z_{1}^{ \pm 1}, z_{2}^{ \pm 1}\right]} & \left(\left(M_{1}\right)_{z_{1}} \otimes\left(M_{2}\right)_{z_{2}}\right) \\
\longrightarrow & \mathbf{k}\left(z_{1}, z_{2}\right) \otimes_{\mathbf{k}\left[z_{1}^{ \pm 1}, z_{2}^{ \pm 1}\right]}\left(\left(M_{2}\right)_{z_{2}} \otimes\left(M_{1}\right)_{z_{1}}\right)
\end{aligned}
$$

satisfying

$$
R_{\left(M_{1}\right)_{z_{1}},\left(M_{2}\right)_{z_{2}}}^{\text {norm }}\left(\left(u_{1}\right)_{z_{1}} \otimes\left(u_{2}\right)_{z_{2}}\right)=\left(u_{2}\right)_{z_{2}} \otimes\left(u_{1}\right)_{z_{1}} .
$$

Note that $\mathbf{k}\left(z_{1}, z_{2}\right) \otimes_{\mathbf{k}\left[z_{1}^{ \pm 1}, z_{2}^{ \pm 1}\right]}\left(\left(M_{1}\right)_{z_{1}} \otimes\left(M_{2}\right)_{z_{2}}\right)$ is a simple $\mathbf{k}\left(z_{1}, z_{2}\right) \otimes U_{q}^{\prime}(\mathfrak{g})$-module ([18, Proposition 9.5]). We call $R_{\left(M_{1}\right)_{z_{1}},\left(M_{2}\right)_{z_{2}}}^{\text {norm }}$ the normalized $R$-matrix.

Let $d_{M_{1}, M_{2}}(u) \in \mathbf{k}[u]$ be a monic polynomial of the smallest degree such that the image of $d_{M_{1}, M_{2}}\left(z_{2} / z_{1}\right) R_{\left(M_{1}\right)_{z_{1}},\left(M_{2}\right)_{z}}^{\text {norm }}$ is contained in $\left(M_{2}\right)_{z_{2}} \otimes\left(M_{1}\right)_{z_{1}}$. We call $d_{M_{1}, M_{2}}(u)$ the denominator of $R_{M_{1}, M_{2}}^{\text {norm }}$. Then,

$$
R_{M_{z_{1}}, N_{z_{2}}}^{\mathrm{ren}}:=d_{M_{1}, M_{2}}\left(z_{2} / z_{1}\right) R_{\left(M_{1}\right)_{z_{1}},\left(M_{2}\right)_{z_{2}}}^{\mathrm{norm}}:\left(M_{1}\right)_{z_{1}} \otimes\left(M_{2}\right)_{z_{2}} \longrightarrow\left(M_{2}\right)_{z_{2}} \otimes\left(M_{1}\right)_{z_{1}}
$$

is a renormalized $R$-matrix, and

$$
\mathbf{r}_{M_{1}, M_{2}}: M_{1} \otimes M_{2} \longrightarrow M_{2} \otimes M_{1}
$$

is equal to the specialization of $R_{M_{z_{1}}, N_{z_{2}}}^{\mathrm{ren}}$ at $z_{1}=z_{2}=1$ up to a constant multiple.

A simple module $V \in \mathscr{C}_{\mathfrak{g}}$ is called real if $V \otimes V$ is simple.

Lemma 1.5.1 ([13]). Let $V, W$ be simple modules in $\mathscr{C}_{\mathfrak{g}}$ and assume that one of them is real. Then

(i) $V \otimes W$ and $W \otimes V$ have simple socles and simple heads. 
(ii) Moreover, $\operatorname{Im}\left(\mathbf{r}_{V, W}\right)$ is equal to the head of $V \otimes W$ and socle of $W \otimes V$.

Similarly to the quiver Hecke algebra case, for $V, W \in \operatorname{Mod}\left(U_{q}^{\prime}(\mathfrak{g})\right)$, we denote by $V \nabla W$ and $V \Delta W$ the head and the socle of $V \otimes W$, respectively.

The following lemma can be proved similarly to the quiver Hecke algebra case ([16, Proposition 3.2.9]), and we do not repeat the proof.

Lemma 1.5.2. Let $V, W$ be simple modules in $\mathscr{C}_{g}$ and assume that one of them is real. Then one has

$$
\operatorname{Hom}(V \otimes W, W \otimes V)=\mathbf{k} \mathbf{r}_{V, W}
$$

1.6. Hernandez-Leclerc's subcategory. For each quantum affine algebra $U_{q}^{\prime}(\mathfrak{g})$, we define a quiver $\mathscr{S}(\mathfrak{g})$ as follows:

(1) we take the set of equivalence classes $\hat{I}_{\mathfrak{g}}:=\left(I_{0} \times \mathbf{k}^{\times}\right) / \sim$ as the set of vertices, where the equivalence relation is given by $(i, x) \sim(j, y)$ if and only if $V\left(\varpi_{i}\right)_{x} \cong V\left(\varpi_{j}\right)_{y}$

(2) we put $d$ many arrows from $(i, x)$ to $(j, y)$, where $d$ denotes the order of zero of $d_{V\left(\varpi_{i}\right), V\left(\varpi_{j}\right)}\left(z_{V\left(\varpi_{j}\right)} / z_{V\left(\varpi_{i}\right)}\right)$ at $z_{V\left(\varpi_{j}\right)} / z_{V\left(\varpi_{i}\right)}=y / x$.

Note that $(i, x)$ and $(j, y)$ are linked by at least one arrow in $\mathscr{S}(\mathfrak{g})$ if and only if the tensor product $V\left(\varpi_{i}\right)_{x} \otimes V\left(\varpi_{j}\right)_{y}$ is reducible ([1, Corollary 2.4]).

Let $\mathscr{S}_{0}(\mathfrak{g})$ be a connected component of $\mathscr{S}(\mathfrak{g})$. Note that a connected component of $\mathscr{S}(\mathfrak{g})$ is unique up to a spectral parameter shift and hence $\mathscr{S}_{0}(\mathfrak{g})$ is uniquely determined up to a quiver isomorphism. Let $\mathscr{C}_{\mathfrak{g}}^{0}$ be the smallest full subcategory of $\mathscr{C}_{\mathfrak{g}}$ stable under taking subquotients, extensions, tensor products and containing $\left\{V\left(\varpi_{i}\right)_{x} ;(i, x) \in \mathscr{S}_{0}(\mathfrak{g})\right\}$. This category for symmetric affine type $\mathfrak{g}$ was introduced in [9]. Note that every simple modules in $\mathscr{C}_{\mathfrak{g}}$ is a tensor product of certain parameter shifts of some simple modules in $\mathscr{C}_{\mathfrak{g}}^{0}\left(\left[9\right.\right.$, Section 3.7]). The Grothendieck ring $K\left(\mathscr{C}_{\mathfrak{g}}^{0}\right)$ of $\mathscr{C}_{\mathfrak{g}}^{0}$ is the polynomial ring generated by the classes of modules in $\left\{V\left(\varpi_{i}\right)_{x} ;(i, x) \in \mathscr{S}_{0}(\mathfrak{g})\right\}$ $([6])$.

1.7. KLR-type quantum affine Schur-Weyl duality functors. In this subsection, we recall the construction of the generalized quantum affine Schur-Weyl duality functor $([11])$.

Let $U_{q}^{\prime}(\mathfrak{g})$ be a quantum affine algebra over $\mathbf{k}$.

Assume that we are given an index set $J$, a family $\left\{V_{j}\right\}_{j \in J}$ of good $U_{q}^{\prime}(\mathfrak{g})$-modules and a map $X: J \rightarrow \mathbf{k}^{\times}$.

We define a quiver $\Gamma^{J}$ associated with the datum $\left(J, X,\left\{V_{j}\right\}_{j \in J}\right)$ as follows:

(1) we take $J$ as the set of vertices,

(2) we put $d_{i j}$ many arrows from $i$ to $j$, where $d_{i j}$ denotes the order of zero of $d_{V_{i}, V_{j}}\left(z_{V_{j}} / z_{V_{i}}\right)$ at $z_{V_{j}} / z_{V_{i}}=X(j) / X(i)$.

Note that we have $d_{i j} d_{j i}=0$ for $i, j \in J$. 
We define a symmetric Cartan matrix $A^{J}=\left(a_{i j}^{J}\right)_{i, j \in J}$ by

$$
a_{i j}^{J}= \begin{cases}2 & \text { if } i=j \\ -d_{i j}-d_{j i} & \text { if } i \neq j .\end{cases}
$$

We give a family of polynomials $\left\{Q_{i, j}(u, v)\right\}_{i, j \in J}$ satisfying (1.1) with the form

$$
Q_{i, j}(u, v)= \pm(u-v)^{-a_{i j}} \quad \text { for } i \neq j
$$

for some choices of sign \pm .

Then we choose a family $\left\{P_{i, j}(u, v)\right\}_{i, j \in J}$ of elements in $\mathbf{k}[[u, v]]$ satisfying the following conditions.

(1) $P_{i, i}(u, v)=1$ for $i \in J$.

(2) The homomorphism $P_{i j}(u, v) R_{\left(V_{i}\right)_{X(i)},\left(V_{j}\right)_{X(j)}}^{\text {norm }}\left(z_{\left(V_{i}\right)_{X(i)}}, z_{\left(V_{j}\right)_{X(j)}}\right)$ has no

$$
\left\{\begin{array}{l}
\text { pole and no zero at } u=v=0 \text {, where } \mathbf{k}\left[z_{\left(V_{i}\right)_{X(i)}}^{ \pm 1}, z_{\left(V_{j}\right)_{X(j)}}^{ \pm 1}\right] \rightarrow \mathbf{k}[[u, v]] \\
\text { is given by } z_{\left(V_{i}\right)_{X(i)}} \mapsto 1+u \text { and } z_{\left(V_{j}\right)_{X(j)}} \mapsto 1+v . \\
(3) Q_{i, j}(u, v)=P_{i, j}(u, v) P_{j, i}(v, u) \text { for } i \neq j .
\end{array}\right.
$$

We call such a family $\left\{P_{i, j}(u, v)\right\}_{i, j \in J}$ a duality coefficient. Note that we have $P_{i, j}(u, v) \in \mathbf{k}[[u, v]]^{\times}(u-v)^{d_{i j}}$.

Let $\left\{\alpha_{i}^{J} ; i \in J\right\}$ be the set of simple roots corresponding to the Cartan matrix $A^{J}$ and $\mathrm{Q}_{J}^{+}=\sum_{i \in J} \mathbb{Z}_{\geq 0} \alpha_{i}^{J}$ be the corresponding positive root lattice. We define the coupling $($,$) by \left(\alpha_{i}^{J}, \alpha^{J}\right)=a_{i j}^{J}$.

Let us denote by $R^{J}(\beta)\left(\beta \in \mathrm{Q}_{J}^{+}\right)$the symmetric quiver Hecke algebra associated with the Cartan matrix $A^{J}$ and the parameter $\left\{Q_{i, j}(u, v)\right\}_{i, j \in J}$.

For each $\nu=\left(\nu_{1}, \ldots, \nu_{n}\right) \in J^{\beta}$, let

$$
\widehat{\mathcal{O}}_{\mathbb{T}^{n}, X(\nu)}=\mathbf{k}\left[\left[X_{1}-X\left(\nu_{1}\right), \ldots, X_{n}-X\left(\nu_{n}\right)\right]\right]
$$

be the completion of the local ring $\mathcal{O}_{\mathbb{T}^{n}, X(\nu)}$ of $\mathbb{T}^{n}$ at $X(\nu):=\left(X\left(\nu_{1}\right), \ldots, X\left(\nu_{n}\right)\right)$. Here $\mathbb{T}=\operatorname{Spec}\left(\mathbf{k}\left[X, X^{-1}\right]\right)$. Set

$$
V_{\nu}=\left(V_{\nu_{1}}\right)_{\mathrm{aff}} \otimes \cdots \otimes\left(V_{\nu_{n}}\right)_{\mathrm{aff}}
$$

Then $V_{\nu}$ is a left $\left(\mathbf{k}\left[X_{1}^{ \pm 1}, \ldots, X_{n}^{ \pm 1}\right] \otimes U_{q}^{\prime}(\mathfrak{g})\right)$-module, where $X_{k}=z_{V_{\nu_{k}}}$. We define

$$
\begin{gathered}
\widehat{V}_{\nu}:=\widehat{\mathcal{O}}_{\mathbb{T}^{n}, X(\nu)} \otimes_{\mathbf{k}\left[X_{1}^{ \pm 1}, \ldots, X_{n}^{ \pm 1}\right]} V_{\nu}, \quad \widehat{V}^{\otimes \beta}:=\bigoplus_{\nu \in J^{\beta}} \widehat{V}_{\nu} e(\nu), \\
\text { and } \widehat{V}_{K}^{\otimes \beta}:=\bigoplus_{\nu \in J^{\beta}} \operatorname{Frac}\left(\widehat{\mathcal{O}}_{\mathbb{T}^{n}, X(\nu)}\right) \otimes_{\mathbf{k}\left[X_{1}^{ \pm 1}, \ldots, X_{n}^{ \pm 1}\right]} V_{\nu},
\end{gathered}
$$

where $\operatorname{Frac}\left(\widehat{\mathcal{O}}_{\mathbb{T}^{n}, X(\nu)}\right)$ denotes the ring of fractions of $\widehat{\mathcal{O}}_{\mathbb{T}^{n}, X(\nu)}$.

Let $e(\nu) \in R^{J}(\beta)$ act on $\widehat{V}^{\otimes \beta}$ as the projection and $x_{k} e(\nu) \in R^{J}(\beta)$ as the multiplication by $X\left(\nu_{k}\right)^{-1}\left(X_{k}-X\left(\nu_{k}\right)\right) e(\nu)$.

Assign $e(\nu) \tau_{a}$ to a k-linear map on $\widehat{V}_{K}^{\otimes \beta}$ defined by

$$
e(\nu) \tau_{a}= \begin{cases}R_{a, a+1}^{\nu} \circ P_{\nu_{a}, \nu_{a+1}}\left(x_{a}, x_{a+1}\right) \circ e(\nu) & \text { if } \nu_{a} \neq \nu_{a+1}, \\ \left(x_{a}-x_{a+1}\right)^{-1} \circ\left(R_{a, a+1}^{\nu}-1\right) \circ e(\nu) & \text { if } \nu_{a}=\nu_{a+1},\end{cases}
$$


where $R_{a, a+1}^{\nu}: \widehat{V}^{\otimes \beta} \rightarrow \widehat{V}_{K}^{\otimes \beta}$ is the k-linear map given by

$$
R_{a, a+1}^{\nu}=\mathrm{id} \otimes \cdots \mathrm{id} \otimes R_{V_{\nu_{a}}, V_{\nu_{a+1}}}^{\mathrm{norm}}\left(z_{V_{\nu_{a}}}, z_{V_{\nu_{a+1}}}\right) \otimes \mathrm{id} \cdots \otimes \mathrm{id} .
$$

The following theorem is one of the main results of [11].

Theorem 1.7.1. The assignments above give a well-defined right action of $R^{J}(\beta)$ on $\widehat{V}^{\otimes \beta}$ which commutes with the left action of $U_{q}^{\prime}(\mathfrak{g})$.

Note that $\widehat{V}^{\otimes \beta}$ is understood to be the trivial $U_{q}^{\prime}(\mathfrak{g})$-module $\mathbf{k}$ when $\beta=0$.

Remark 1.7.2. The action of $R^{J}(\beta)$ on $\widehat{V}^{\otimes \beta}$ depends on the choice of duality coefficients. In [11], we take $P_{i, j}(u, v)=(u-v)^{d_{i j}}$ as the duality coefficient. However, the same proof still works for the above theorem with an arbitrary choice of duality coefficients.

For each $\beta \in \mathbf{Q}_{J}^{+}$, we define the functor

$$
\mathcal{F}_{\beta}: \operatorname{Mod}\left(R^{J}(\beta)\right) \rightarrow \operatorname{Mod}\left(U_{q}^{\prime}(\mathfrak{g})\right)
$$

by

$$
\mathcal{F}_{\beta}(M):=\widehat{V}^{\otimes \beta} \otimes_{R^{J}(\beta)} M,
$$

where $M$ is an $R^{J}(\beta)$-module.

Set

$$
\mathcal{F}:=\bigoplus_{\beta \in Q_{J}^{+}} \mathcal{F}_{\beta}: \bigoplus_{\beta \in Q_{J}^{+}} \operatorname{Mod}\left(R^{J}(\beta)\right) \rightarrow \operatorname{Mod}\left(U_{q}^{\prime}(\mathfrak{g})\right)
$$

Note that the functor $\mathcal{F}$ depends on the choice of the duality coefficient $\left\{P_{i, j}(u, v)\right\}_{i, j \in J}$.

Theorem 1.7.3 ([11]). If the Cartan matrix $A^{J}$ associated with $R^{J}$ is of finite type $A, D$ or $E$, then the functor $\mathcal{F}_{\beta}$ is exact for every $\beta \in \mathrm{Q}_{J}^{+}$.

For each $i \in J$, let $L(i)$ be the 1-dimensional $R^{J}\left(\alpha_{i}^{J}\right)$-module generated by a nonzero vector $u(i)$ with relation $x_{1} u(i)=0$ and $e(j) u(i)=\delta(j=i) u(i)$ for $j \in J$. Then the affinization $L(i)_{z}:=\mathbf{k}[z] \otimes L(i)$ is isomorphic to $R^{J}\left(\alpha_{i}^{J}\right)$ as a left $R^{J}\left(\alpha_{i}^{J}\right)$-module.

By the construction, we have

Proposition 1.7.4. ([11, Proposition 3.2.2])

(i) For any $i \in J$, we have

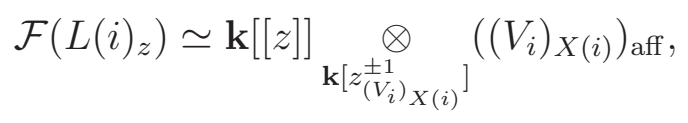

where $\mathbf{k}\left[z_{\left(V_{i}\right)_{X(i)}}^{ \pm 1}\right] \rightarrow \mathbf{k}[[z]]$ is given by $z_{\left(V_{i}\right)_{X(i)}} \mapsto 1+z$. In particular, we have

$$
\mathcal{F}(L(i)) \simeq\left(V_{i}\right)_{X(i)} \quad \text { for } i \in J
$$


(ii) For $i, j \in J$, let

$$
\phi=R_{L(i)_{z}, L(j)_{z^{\prime}}}: L(i)_{z} \circ L(j)_{z^{\prime}} \rightarrow L(j)_{z^{\prime}} \circ L(i)_{z} .
$$

That is, let $\phi$ be the $R^{J}\left(\alpha^{J_{i}}+\alpha^{J}{ }_{j}\right)$-module homomorphism given by

$$
\phi\left(u(i)_{z} \otimes u(j)_{z^{\prime}}\right)=\varphi_{1}\left(u(j)_{z^{\prime}} \otimes u(i)_{z}\right),
$$

where $\varphi_{1}$ is the intertwiner in (1.3). Then we have

$$
\mathcal{F}(\phi)=P_{i, j}\left(z, z^{\prime}\right) R_{\mathcal{F}(L(i)), \mathcal{F}(L(j))}^{\text {norm }}\left(z_{\mathcal{F}(L(i))}, z_{\mathcal{F}(L(j))}\right)
$$

as a $U_{q}^{\prime}(\mathfrak{g})$-module homomorphism

$$
\begin{aligned}
& \mathbf{k}\left[\left[z, z^{\prime}\right]\right]_{\mathbf{k}\left[z_{\mathcal{F}(L(i))}^{ \pm 1}, z_{\mathcal{F}(L(j))}^{ \pm 1}\right]}\left(\mathcal{F}(L(i))_{\text {aff }} \otimes \mathcal{F}(L(j))_{\text {aff }}\right) \\
& \longrightarrow \mathbf{k}\left[\left[z, z^{\prime}\right]\right] \otimes_{\mathbf{k}\left[z_{\mathcal{F}(L(i))}^{ \pm 1}, z_{\mathcal{F}(L(j))}^{ \pm 1}\right]}\left(\mathcal{F}(L(j))_{\text {aff }} \otimes \mathcal{F}(L(i))_{\text {aff }}\right)
\end{aligned}
$$

where $\mathbf{k}\left[z_{\mathcal{F}(L(i))}^{ \pm 1}, z_{\mathcal{F}(L(j))}^{ \pm 1}\right] \rightarrow \mathbf{k}\left[\left[z, z^{\prime}\right]\right]$ is given by $z_{\mathcal{F}(L(i))} \mapsto 1+z$ and $z_{\mathcal{F}(L(j))} \mapsto$ $1+z^{\prime}$.

Recall that $\mathscr{C}_{\mathfrak{g}}$ denotes the category of finite-dimensional integrable $U_{q}^{\prime}(\mathfrak{g})$-modules.

Theorem 1.7.5. ([11]) The functor $\mathcal{F}$ induces a tensor functor

$$
\mathcal{F}: \bigoplus_{\beta \in Q_{J}^{+}} R^{J}(\beta) \text { gmod } \rightarrow \mathscr{C}_{\mathfrak{g}} .
$$

Namely, $\mathcal{F}$ sends finite-dimensional graded $R^{J}(\beta)$-modules to $U_{q}^{\prime}(\mathfrak{g})$-modules in $\mathscr{C}_{\mathfrak{g}}$, and there exist canonical $U_{q}^{\prime}(\mathfrak{g})$-module isomorphisms

$$
\mathcal{F}\left(R^{J}(0)\right) \simeq \mathbf{k}, \quad \mathcal{F}\left(M_{1} \circ M_{2}\right) \simeq \mathcal{F}\left(M_{1}\right) \otimes \mathcal{F}\left(M_{2}\right)
$$

for $M_{1} \in R^{J}\left(\beta_{1}\right)$-gmod and $M_{2} \in R^{J}\left(\beta_{2}\right)$-gmod such that the diagrams in [11, A.1.2] are commutative .

The following theorem can be proved in a similar way as in [20, Theorem 4.1] and we omit the proof.

Theorem 1.7.6. Assume that the functor $\mathcal{F}$ in Theorem 1.7 .5 is exact.

(i) For any simple module $M$ in $R^{J}(\beta)$-gmod, $\mathcal{F}(M)$ is either a simple module or a zero module. In particular, if $M$ is a real simple module and $\mathcal{F}(M)$ is non-zero, then $\mathcal{F}(M)$ is a real simple module.

(ii) Let $M$ and $N$ be simple modules in $R^{J}$-gmod, and assume that one of them is real. Then $\mathcal{F}(M \nabla N)$ is zero or isomorphic to $\mathcal{F}(M) \nabla \mathcal{F}(N)$.

Hence if the functor $\mathcal{F}$ is exact, then $\mathcal{F}$ induces a surjective map from a subset of the isomorphism classes of simple subquotients of a module $M \in R^{J}$-gmod to the set of the isomorphism classes of simple subquotients of $\mathcal{F}(M)$. Hence we have the following corollary. 
Corollary 1.7.7. Assume that the functor $\mathcal{F}$ in Theorem 1.7 .5 is exact and assume that $M_{1}, M_{2}, \ldots, M_{r}$ are modules in $R^{J}$-gmod. Then, for any simple subquotient $V$ of $\mathcal{F}\left(M_{1}\right) \otimes F\left(M_{2}\right) \otimes \cdots \otimes \mathcal{F}\left(M_{r}\right)$, there exists a simple subquotient $L$ of $M_{1} \circ M_{2} \circ \cdots \circ M_{r}$ such that $V \simeq \mathcal{F}(L)$.

Assume that the functor $\mathcal{F}$ in Theorem 1.7.5 is exact. Let us denote by $\mathscr{C}_{J}$ the full subcategory of $\mathscr{C}_{\mathfrak{g}}$ consisting of $V$ such that every composition factor of $V$ appears as a composition factor of a tensor product of modules of the form $\left(V_{i}\right)_{X(i)} \simeq \mathcal{F}(L(i))$ $(i \in J)$. In other words, $\mathscr{C}_{J}$ is the smallest abelian subcategory of $\mathscr{C}_{\mathfrak{g}}$ which contains all $\left(V_{i}\right)_{X(i)}$ 's, and is stable under taking submodules, quotients, extensions and tensor products. Hence we have

$$
\mathcal{F}: \bigoplus_{\beta \in Q_{J}^{+}} R^{J}(\beta) \text {-gmod } \rightarrow \mathscr{C}_{J} \subset \mathscr{C}_{\mathfrak{g}}
$$

The following lemma will be used in the next section.

Lemma 1.7.8. Assume that $\mathcal{F}$ is exact. Let $M, N \in R^{J}$-gmod be simple modules, and assume that one of them is real. We assume further that $\mathfrak{D}(M, N) \leq 1$. Assume that $\mathcal{F}(M), \mathcal{F}(N) \neq 0$ and $\mathcal{F}(M) \otimes \mathcal{F}(N)$ is not simple. Then the homomorphism $\mathcal{F}\left(\mathbf{r}_{M, N}\right)$ is equal to $\mathbf{r}_{\mathcal{F}(M), \mathcal{F}(N)}$ up to a non-zero constant multiple, and we have $\mathcal{F}(M \nabla N) \simeq$ $\mathcal{F}(M) \nabla \mathcal{F}(N)$. In particular, it is a simple module.

Proof. If $\mathfrak{D}(M, N)=0$, then $\mathcal{F}(M) \otimes \mathcal{F}(N)$ is simple, and we need nothing to prove. Assume that $\mathfrak{D}(M, N)=1$. Then we have exact sequences (ignoring grading shifts)

$$
\begin{gathered}
0 \rightarrow N \nabla M \rightarrow M \circ N \rightarrow M \nabla N \rightarrow 0, \\
0 \rightarrow M \nabla N \rightarrow N \circ M \rightarrow N \nabla M \rightarrow 0
\end{gathered}
$$

by Lemma 1.3.5. Applying the exact functor $\mathcal{F}$, we obtain an exact sequence

$$
0 \rightarrow \mathcal{F}(N \nabla M) \rightarrow \mathcal{F}(M) \otimes \mathcal{F}(N) \rightarrow \mathcal{F}(M \nabla N) \rightarrow 0
$$

Note that $\mathcal{F}(N \nabla M)$ and $\mathcal{F}(M \nabla N)$ are simple or zero. Since the length of $\mathcal{F}(M) \otimes \mathcal{F}(N)$ is at least two by the assumption, we conclude that $\mathcal{F}(N \nabla M)$ and $\mathcal{F}(M \nabla N)$ are simple modules. Hence $\mathcal{F}\left(\mathbf{r}_{M, N}\right)$ which is the composition $\mathcal{F}(M) \otimes \mathcal{F}(N) \rightarrow \mathcal{F}(M \nabla N) \longmapsto$ $\mathcal{F}(N) \otimes \mathcal{F}(M)$, does not vanish. Hence it is equal to $\mathbf{r}_{\mathcal{F}(M), \mathcal{F}(N))}$ up to a non-zero constant multiple by Lemma 1.5.2.

\section{Quantum affine Algebra of type B And Duality Functors}

In this section, we will construct and study a generalized quantum affine Schur Weyl duality functor whose codomain is the module category of quantum affine algebra of type $B$.

2.1. Quantum affine algebra of type $\mathbf{B}_{n}^{(1)}$. We recall the quantum affine algebra of type $B_{n}^{(1)}$ and fix the conventions. From now on, we fix $n \geq 2$ and set $N=2 n$. Let $\mathfrak{g}$ be the affine Kac-Moody algebra of type $B_{n}^{(1)}$. The index set of simple roots is given by $I=\{0,1, \ldots, n\}$ and we have $I_{0}=\{1,2, \ldots, n\}$. The Dynkin diagrams and the fundamental weights are given as follows: 


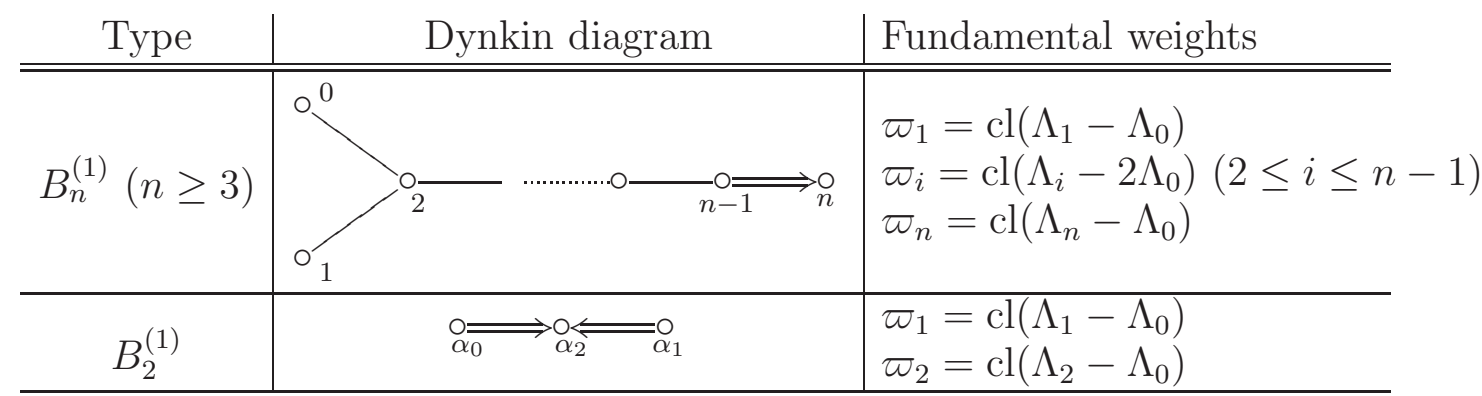

Note that the Dynkin diagram of type $B_{2}^{(1)}$ in the table above is denoted by $C_{2}^{(1)}$ in [10].

By (1.7), We have

$$
\left(\alpha_{i}, \alpha_{i}\right)= \begin{cases}2 & \text { if } i=0,1, \ldots, n-1 \\ 1 & \text { if } i=n\end{cases}
$$

We set

$$
q_{s}:=q_{n}=q^{1 / 2} \quad \text { and } \quad q_{t}:=(-1)^{n+1} q_{s}^{2 n+1} .
$$

The null root and the canonical central element are given by

$$
\begin{aligned}
& \delta=\alpha_{0}+\alpha_{1}+2\left(\alpha_{2}+\cdots+\alpha_{n-1}+\alpha_{n}\right), \\
& c=h_{0}+h_{1}+2\left(h_{2}+\cdots+h_{n-1}\right)+h_{n} .
\end{aligned}
$$

It follows that

$$
p^{*}=q^{2 n-1} .
$$

The fundamental representations of $U_{q}^{\prime}\left(B_{n}^{(1)}\right)$ are of the form $V\left(\varpi_{i}\right)_{x}$ with $1 \leq i \leq n$ and $x \in \mathbf{k}^{\times}$. For $k>n+1$ or $k<0, V\left(\varpi_{k}\right)$ is understood to be zero, and the modules $V\left(\varpi_{0}\right)$ and $V\left(\varpi_{n+1}\right)$ are understood to be the trivial representation.

Recall that $d_{V\left(\varpi(i), V\left(\varpi_{j}\right)\right.}(z)$ denotes the denominator of the normalized R-matrix $R_{V\left(\varpi_{i}\right), V\left(\varpi_{j}\right)_{z}}^{\text {norm }}$ The following formula of the denominators are given in [23].

Proposition 2.1.1 ([23]). For $1 \leq k, l \leq n-1$, we have

$$
d_{V\left(\varpi_{k}\right), V\left(\varpi_{l}\right)}(z)=\prod_{s=1}^{\min (k, l)}\left(z-(-1)^{k+l} q^{|k-l|+2 s}\right)\left(z-(-1)^{k+l} q^{2 n-k-l-1+2 s}\right) .
$$

For $1 \leq k \leq n-1$, we have

$$
d_{V\left(\varpi_{k}\right), V\left(\varpi_{n}\right)}(z)=\prod_{s=1}^{k}\left(z-(-1)^{n+k} q_{s}^{2 n-2 k-1+4 s}\right) .
$$

Finally, we have

$$
d_{V\left(\varpi_{n}\right), V\left(\varpi_{n}\right)}(z)=\prod_{s=1}^{n}\left(z-\left(q_{s}\right)^{4 s-2}\right) .
$$


Note that $d_{V\left(\varpi_{i}\right), V\left(\varpi_{j}\right)}(z)=d_{V\left(\varpi_{j}\right), V\left(\varpi_{i}\right)}(z)$.

Proposition 2.1.2 ([23]). There exist surjective $U_{q}^{\prime}\left(B_{n}^{(1)}\right)$-module homomorphisms (2.6) $V\left(\varpi_{k}\right)_{(-q)^{-l}} \otimes V\left(\varpi_{l}\right)_{(-q)^{k}} \rightarrow V\left(\varpi_{k+l}\right)$ for $1 \leq k, l \leq n-1$ with $k+l \leq n-1$,

$$
\begin{aligned}
& V\left(\varpi_{n}\right)_{q^{k+1}} \otimes V\left(\varpi_{n}\right)_{q^{N-k}} \rightarrow V\left(\varpi_{k}\right)_{(-1)^{k+1} q_{t}} \text { for } 1 \leq k \leq n-1, \\
& V\left(\varpi_{n}\right) \otimes V\left(\varpi_{1}\right)_{q_{t}} \rightarrow V\left(\varpi_{n}\right)_{q^{2}} .
\end{aligned}
$$

2.2. The quiver $\Gamma$. Recall that $\mathscr{C}_{B_{n}^{(1)}}^{(1)}$ is the smallest full subcategory of $\mathscr{C}_{B_{n}^{(1)}}$ stable un-

der taking subquotients, extensions, tensor products and containing $\left\{V\left(\varpi_{i}\right)_{x} ;(i, x) \in \mathscr{S}_{0}\left(B_{n}^{(1)}\right)\right\}$. Here we take the following connected component $\mathscr{S}_{0}\left(B_{n}^{(1)}\right)$ of $\mathscr{S}\left(B_{n}^{(1)}\right)$ :

$$
\mathscr{S}_{0}\left(B_{n}^{(1)}\right):=\left\{\left(i,(-1)^{i-1} q_{t} q^{m}\right),\left(n, q^{m}\right) ; 1 \leq i \leq n-1, m \in \mathbb{Z}\right\} .
$$

Let $J=\mathbb{Z}$, and define

$$
V_{j}:= \begin{cases}V\left(\varpi_{n}\right) & \text { if } j \equiv-1,0 \bmod N \\ V\left(\varpi_{1}\right) & \text { otherwise. }\end{cases}
$$

for $j \in J$. The map $X: J \rightarrow \mathbf{k}^{\times}$is given as follows:

$$
\begin{aligned}
& X(0)=q^{0}=1 \\
& X(j)=q_{t} q^{2(j-1)} \quad(1 \leq j \leq N-2), \\
& X(N-1)=q^{3 N-5}
\end{aligned}
$$

and we extend it by

$$
X(j+k N):=X(j) q^{k(2 N-2)}=X(j) p^{* 2 k} \quad \text { for } 0 \leq j \leq N-1 \text { and } k \in \mathbb{Z} .
$$

Then by Proposition 2.1.1 we have

$$
d_{i j}=\delta(j=i+1, j \not \equiv 0 \bmod N)+\delta(j=i-1, j \equiv-1 \bmod N) .
$$

For $i, j \in J$, we have

$$
\left(\alpha_{i}^{J}, \alpha_{j}^{J}\right)= \begin{cases}-1 & \text { if } i-j= \pm 1, \\ 2 & \text { if } i=j, \\ 0 & \text { otherwise. }\end{cases}
$$

Hence the quiver $\Gamma=\Gamma^{J}$ corresponding to the datum $\left(J, X,\left\{V_{j}\right\}_{j \in J}\right)$ and the corresponding quiver Hecke algebra are of type $A_{\infty}$.

We give the parameters for $R^{J}$ by

$$
Q_{i, j}(u, v)= \begin{cases} \pm(u-v) & \text { if } j=i \pm 1 \\ 0 & \text { if } i=j \\ 1 & \text { otherwise }\end{cases}
$$


Remark 2.2.1. The above choice of parameters $\left\{Q_{i, j}(u, v)\right\}_{i j \in J}$ for quiver Hecke algebra $R$ of type $A_{\infty}$ is identical with those in [11] and [14].

Let us choose a duality coefficient $\left\{P_{i, j}(u, v) \in \mathbf{k}[[u, v]] ; i, j \in J\right\}$ satisfying condition (1.17) including

$$
Q_{i, j}(u, v)=P_{i, j}(u, v) P_{j, i}(v, u) \quad \text { for } i \neq j .
$$

For example, we may take

$$
P_{i, j}^{\mathrm{T}}(u, v):=(-1)^{\delta(j=i+1 \equiv 0 \bmod N)}(u-v)^{d_{i j}} .
$$

as $P_{i, j}(u, v)$.

We take

$$
P_{J}=\bigoplus_{a \in \mathbb{Z}} \mathbb{Z} \epsilon_{a}
$$

as the weight lattice with $\left(\epsilon_{a}, \epsilon_{b}\right)=\delta_{a, b}$. The root lattice $Q_{J}=\bigoplus_{i \in J} \mathbb{Z} \alpha_{i}^{J}$ is embedded into $P_{J}$ by $\alpha_{i}^{J}=\epsilon_{i}-\epsilon_{i+1}$. We write $\mathrm{Q}_{J}^{+}$for $\bigoplus_{i \in J} \mathbb{Z}_{\geq 0} \alpha_{i}^{J}$.

Then we have a family of functors $\mathcal{F}_{\beta}\left(\beta \in Q_{J}^{+}\right)$defined in (1.19). Note that $\mathcal{F}_{\beta}$ depends on the choice of duality coefficient $\left\{P_{i, j}(u, v)\right\}_{i, j \in J}$.

By Theorem 1.7.3, the functor

$$
\mathcal{F}=\bigoplus_{\beta \in Q_{J}^{+}} \mathcal{F}_{\beta}: \bigoplus_{\beta \in Q_{J}^{+}} \operatorname{Mod}_{\mathrm{gr}}\left(R^{J}(\beta)\right) \rightarrow \operatorname{Mod}\left(U_{q}^{\prime}\left(B_{n}^{(1)}\right)\right)
$$

is exact.

Note that

$$
\mathcal{F}(L(a+N)) \simeq{ }^{* *} \mathcal{F}(L(a)) \text { and } \quad \mathcal{F}(L(a-N)) \simeq \mathcal{F}(L(a))^{* *}
$$

for all $a \in J$ by the construction.

2.3. Simple modules over quiver Hecke algebra of type $\mathbf{A}_{\infty}$. Since we deal with a functor $\mathcal{F}$ whose domain is the category of modules over the quiver Hecke algebra of type $A_{\infty}$, we recall some basic materials for the quiver Hecke algebra of type $A_{\infty}$.

For simplicity we will write $R(\beta)$ for $R^{J}(\beta)$ in the sequel. A pair of integers $(a, b)$ such that $a \leq b$ is called a segment. The length of $(a, b)$ is $b-a+1$. A multisegment is a finite sequence of segments.

For a segment $(a, b)$ of length $\ell$, we define a graded 1-dimensional $R\left(\epsilon_{a}-\epsilon_{b+1}\right)$-module $L(a, b)=\mathbf{k} u(a, b)$ in $R\left(\epsilon_{a}-\epsilon_{b+1}\right)$-gmod which is generated by a vector $u(a, b)$ of degree 0 with the action of $R\left(\epsilon_{a}-\epsilon_{b+1}\right)$ given by

$$
x_{m} u(a, b)=0, \quad \tau_{k} u(a, b)=0, \quad e(\nu) u(a, b)= \begin{cases}u(a, b) & \text { if } \nu=(a, a+1, \ldots, b), \\ 0 & \text { otherwise }\end{cases}
$$

We understand that $L(a, a-1)$ is the 1-dimensional module over $R(0)=\mathbf{k}$ and the length of $(a, a-1)$ is 0 . Note that $L(a, a)$ is nothing but $L(a)$.

We give a total order on the set of segments as follows:

$$
\left(a_{1}, b_{1}\right)>\left(a_{2}, b_{2}\right) \quad \text { if } a_{1}>a_{2} \text { or } a_{1}=a_{2} \text { and } b_{1}<b_{2} \text {. }
$$


Then we have

Proposition 2.3.1 ([17, Theorem 4.8, Theorem 5.1], [11, Proposition 4.2.7])

(i) Let $M$ be a simple module in $R(\beta) \operatorname{gmod}$ with $\beta \in \mathrm{Q}_{J}^{+}$. Then there exists a unique pair of a multisegment $\left(\left(a_{1}, b_{1}\right), \ldots,\left(a_{t}, b_{t}\right)\right)$ and an integer $c$ such that

(a) $\left(a_{k}, b_{k}\right) \geq\left(a_{k+1}, b_{k+1}\right)$ for $1 \leq k \leq t-1$,

(b) $\sum_{k=1}^{t}\left(\epsilon_{a_{k}}-\epsilon_{b_{k}+1}\right)=\beta$,

(c) $M \simeq q^{c} \mathrm{hd}\left(L\left(a_{1}, b_{1}\right) \circ \cdots \circ L\left(a_{t}, b_{t}\right)\right)$, where hd denotes the head.

(ii) Conversely, if a multisegment $\left(\left(a_{1}, b_{1}\right), \ldots,\left(a_{t}, b_{t}\right)\right)$ satisfies (a) and (b), then $\operatorname{hd}\left(L\left(a_{1}, b_{1}\right) \circ \cdots \circ L\left(a_{t}, b_{t}\right)\right)$ is a simple $R(\beta)$-module.

If a multisegment $\left(\left(a_{1}, b_{1}\right), \ldots,\left(a_{t}, b_{t}\right)\right)$ satisfies the condition (a) above, then we say that it is an ordered multisegment. We call the ordered multisegment $\left(\left(a_{k}, b_{k}\right)\right)_{1 \leq k \leq t}$ in Proposition 2.3.1 (i) the multisegment associated with $M$.

Proposition 2.3.2 ([11, Proposition 4.2.3]). For $a \leq b$ and $a^{\prime} \leq b^{\prime}$, set $\beta=\epsilon_{a}-\epsilon_{b+1}$ and $\beta^{\prime}=\epsilon_{a^{\prime}}-\epsilon_{b^{\prime}+1}$.

(i) $L(a, b)$ is a real simple module.

(ii) We have

$$
\Lambda\left(L(a, b), L\left(a^{\prime}, b^{\prime}\right)\right)= \begin{cases}-\delta_{a, a^{\prime}}-\delta_{b, b^{\prime}}+2 & \text { if } a \leq a^{\prime} \leq b \leq b^{\prime} \\ -\left(\beta, \beta^{\prime}\right) & \text { otherwise. }\end{cases}
$$

(iii) We have

$$
\begin{aligned}
& \text { D }\left(L(a, b), L\left(a^{\prime}, b^{\prime}\right)\right) \\
& \quad= \begin{cases}0 & \text { if }[a, b] \subset\left[a^{\prime}, b^{\prime}\right] \text { or }[a, b] \supset\left[a^{\prime}, b^{\prime}\right] \text { or }[a-1, b+1] \cap\left[a^{\prime}, b^{\prime}\right]=\emptyset, \\
1 & \text { otherwise. }\end{cases}
\end{aligned}
$$

(iv) If $a^{\prime}<a \leq b^{\prime}<b$, then we have the following exact sequence

$$
\begin{aligned}
0 \longrightarrow q L\left(a^{\prime}, b\right) \circ L\left(a, b^{\prime}\right) \longrightarrow L(a, b) \circ L\left(a^{\prime}, b^{\prime}\right) \\
\stackrel{\mathrm{r}_{L(a, b), L\left(a^{\prime} b^{\prime}\right)}}{\longrightarrow} L\left(a^{\prime}, b^{\prime}\right) \circ L(a, b) \longrightarrow q^{-1} L\left(a^{\prime}, b\right) \circ L\left(a, b^{\prime}\right) \longrightarrow 0 .
\end{aligned}
$$

(v) If $a=b^{\prime}+1$, then we have an exact sequence

$0 \rightarrow q L\left(a^{\prime}, b\right) \longrightarrow L(a, b) \circ L\left(a^{\prime}, b^{\prime}\right) \stackrel{\mathbf{r}_{L(a, b), L\left(a^{\prime} b^{\prime}\right)}}{\longrightarrow} q^{-1} L\left(a^{\prime}, b^{\prime}\right) \circ L(a, b) \rightarrow q^{-1} L\left(a^{\prime}, b\right) \rightarrow 0$.

2.4. Properties of the functor $\mathcal{F}$. In this subsection we will investigate properties of the exact functor

$$
\mathcal{F}: \bigoplus_{\beta \in Q_{J}^{+}} R(\beta) \operatorname{gmod} \rightarrow \mathscr{C}_{J} \subset \mathscr{C}_{B_{n}^{(1)}}
$$

Lemma 2.4.1. We have

$$
\mathcal{F}(L(0, k)) \simeq \begin{cases}V\left(\varpi_{n}\right)_{q^{2 k}} & \text { for } 0 \leq k \leq N-2, \\ \mathbf{k} & \text { for } k=N-1\end{cases}
$$


and

$$
\mathcal{F}(L(k, N-1)) \simeq V\left(\varpi_{n}\right)_{q^{N-3+2 k}} \quad \text { for } 1 \leq k \leq N-1 .
$$

Proof. When $k=0$, isomorphism (2.12) is obvious from the definition. Let $k \geq 1$. By induction on $k$, we may assume that $\mathcal{F}(L(0, k-1)) \simeq V\left(\varpi_{n}\right)_{q^{2(k-1)}}$.

On the other hand, Proposition 2.1.1 implies that

$$
\begin{aligned}
\mathcal{F}(L(0, k-1)) \nabla \mathcal{F}(L(k)) & \\
\simeq & \begin{cases}V\left(\varpi_{n}\right)_{q^{2(k-1)}} \nabla V\left(\varpi_{1}\right)_{q_{t} q^{2 k-2}} \simeq V\left(\varpi_{n}\right)_{q^{2 k}} & \text { for } 1 \leq k \leq N-2, \\
V\left(\varpi_{n}\right)_{q^{2(N-2)}} \nabla V\left(\varpi_{n}\right)_{q^{3 N-5}} \simeq \mathbf{k} & \text { if } k=N-1 .\end{cases}
\end{aligned}
$$

Hence the tensor product $\mathcal{F}(L(0, k-1)) \otimes \mathcal{F}(L(k))$ is not simple for $1 \leq k \leq N-1$, and Lemma 1.7.8 implies that $\mathcal{F}(L(0, k)) \simeq \mathcal{F}(L(0, k-1) \nabla L(k)) \simeq \mathcal{F}(L(0, \bar{k}-1)) \nabla$ $\mathcal{F}(L(k))$. Thus we obtain $(2.12)$.

Let $1 \leq k \leq N-1$. Then, an epimorphism

$$
\begin{aligned}
V\left(\varpi_{n}\right)_{q^{2 k-2}} \otimes \mathcal{F}(L(k, N-1)) & \simeq \mathcal{F}(L(0, k-1) \circ L(k, N-1)) \\
& \rightarrow \mathcal{F}(L(0, k-1) \nabla L(k, N-1)) \simeq \mathcal{F}(0, N-1) \simeq \mathbf{k},
\end{aligned}
$$

induces a non-zero homomorphism $\mathcal{F}(L(k, N-1)) \rightarrow{ }^{*}\left(V\left(\varpi_{n}\right)_{q^{2 k-2}}\right)$. Since $\mathcal{F}(L(k, N-$ 1)) is zero or simple, we obtain

$$
\mathcal{F}(L(k, N-1)) \simeq{ }^{*}\left(V\left(\varpi_{n}\right)_{q^{2 k-2}}\right) \simeq V\left(\varpi_{n}\right)_{q^{2 k-2} p^{*}}=V\left(\varpi_{n}\right)_{q^{N-3+2 k}} .
$$

Since $\left(V_{j}\right)_{X(j)}(j \in J)$ belongs to $\mathscr{C}_{B_{n}^{(1)}}^{0}$, the category $\mathscr{C}_{J}$ is a full subcategory of $\mathscr{C}_{B_{n}^{(1)}}^{0}$. The next proposition asserts that they are actually the same.

Proposition 2.4.2. For any $(i, x) \in \mathscr{S}_{0}\left(B_{n}^{(1)}\right)$, the fundamental representation $V\left(\varpi_{i}\right)_{x}$ belongs to $\mathscr{C}_{J}$. In particular, we have $\mathscr{C}_{J}=\mathscr{C}_{B_{n}^{(1)}}^{(1)}$.

Proof. By Lemma 2.4.1, the fundamental representation $V\left(\varpi_{n}\right)_{q^{m}}$ is in the image of the functor $\mathcal{F}$ if $m \in 2 \mathbb{Z}$ and $0 \leq m \leq 2 N-4$ or if $m \in 2 \mathbb{Z}+1$ and $N-1 \leq m \leq 3 N-5$.

By the relation $X(a+k N)=X(a) q^{k(2 N-2)}$, the category $\mathscr{C}_{J}$ contains the fundamental representations $V\left(\varpi_{n}\right)_{q^{m}}$ for all $m \in \mathbb{Z}$.

By (2.7) we have an epimorphism

$$
V\left(\varpi_{n}\right)_{q^{1+i+m}} \otimes V\left(\varpi_{n}\right)_{q^{N-i+m}} \rightarrow V\left(\varpi_{i}\right)_{(-1)^{i+1} q_{t} q^{m}}
$$

for $1 \leq i \leq n-1$ and $m \in \mathbb{Z}$. Hence $\mathscr{C}_{J}$ contains the other fundamental representations in $\mathscr{C}_{B_{n}^{(1)}}^{(\overline{0)}}$.

By Corollary 1.7.7, we have the following

Corollary 2.4.3. For any simple module $V$ in $\mathscr{C}_{B_{n}^{(1)}}^{(1)}$, there exists a simple module $L \in R^{J}$-gmod such that $\mathcal{F}(L) \simeq V$.

Thus we have 
Corollary 2.4.4. The functor $\mathcal{F}$ induces a surjective ring homomorphism

$$
\phi_{\mathcal{F}}: K\left(R^{J} \text {-gmod }\right) \rightarrow K\left(\mathscr{C}_{B_{n}^{(1)}}^{0}\right)
$$

Remark 2.4.5. Recall that a functor $F: \mathcal{C} \rightarrow \mathcal{C}^{\prime}$ is essentially surjective if, for any $M^{\prime} \in \mathcal{C}^{\prime}$, there exists $M \in \mathcal{C}$ such that $F(M) \simeq M^{\prime}$. We do not know if $\mathcal{F}: R^{J}$-gmod $\rightarrow$ $\mathscr{C}_{B_{n}^{(1)}}^{0}$ is essentially surjective or not.

Proposition 2.4.6. Let $(a, b)$ be a segment with length $\ell:=b-a+1$. Then $\mathcal{F}(L(a, b))$ is non-zero if and only if $\ell \leq N$. Moreover we have

$$
\mathcal{F}(L(a, b)) \simeq \mathbf{k} \quad \text { if } \ell=b-a+1=N .
$$

Proof. In the course of the proof, we omit the gradings.

Assume that $\mathcal{F}(L(a, a+N-1)) \simeq \mathbf{k}$ for some $a \in \mathbb{Z}$. Then we have a homomorphism

$$
\mathcal{F}(L(a)) \otimes \mathcal{F}(L(a+1, a+N-1)) \rightarrow \mathcal{F}(L(a, a+N-1)) \simeq \mathbf{k} .
$$

Hence $\mathcal{F}(L(a+1, a+N-1)$ is a right dual of $\mathcal{F}(L(a))$. It follows that $\mathcal{F}(L(a+N)) \simeq$ ** $\mathcal{F}(L(a))$ is a right dual of $\mathcal{F}(L(a+1, a+N-1)$. Since $L(a+1, a+N)=L(a+1, a+$ $N-1) \nabla L(a+N)$, applying Lemma 1.7.8, we have

$$
\mathcal{F}(L(a+1, a+N)) \simeq \mathcal{F}(L(a+1, a+N-1)) \nabla \mathcal{F}(L(a+N)) \simeq \mathbf{k} .
$$

A similar argument shows that

$$
\mathcal{F}(L(a-1, a+N-2)) \simeq \mathcal{F}(L(a-1)) \nabla \mathcal{F}(L(a+1, a+N-1)) \simeq \mathbf{k} .
$$

Combining it with $(2.12)$ we conclude that $\mathcal{F}(L(a, a+N-1)) \simeq \mathbf{k}$ for all $a \in \mathbb{Z}$.

Assume that $\ell=b-a+1<N$. Then there exists a surjective homomorphism

$$
\mathcal{F}(L(a, b)) \otimes \mathcal{F}(L(b+1, a+N-1)) \rightarrow \mathcal{F}(L(a, a+N-1)) \simeq \mathbf{k}
$$

and hence $\mathcal{F}(L(a, b)) \not 20$.

Note that

$$
\mathcal{F}(L(a)) \simeq \mathcal{F}(L(a)) \otimes \mathcal{F}(L(a+1, a+N-1)) \rightarrow \mathcal{F}(L(a, a+N))
$$

and

$$
\mathcal{F}(L(a+N)) \simeq \mathcal{F}(L(a, a+N-1)) \otimes \mathcal{F}(L(a+N)) \rightarrow \mathcal{F}(L(a, a+N))
$$

for all $a \in \mathbb{Z}$. If $\mathcal{F}(L(a, a+N))$ is a non-zero module, then we have

$$
\mathcal{F}(L(a)) \simeq \mathcal{F}(L(a, a+N)) \simeq \mathcal{F}(L(a+N)) \simeq \mathcal{F}(L(a))_{q^{2 N-2}},
$$

which is a contradiction. Hence we conclude that $\mathcal{F}(L(a, a+N)) \simeq 0$ for all $a \in \mathbb{Z}$. Now assume that $\ell=b-a+1 \geq N+2$. Then there exists a surjective homomorphism

$$
0 \simeq \mathcal{F}(L(a, a+N)) \otimes \mathcal{F}(L(a+N+1, b)) \rightarrow \mathcal{F}(L(a, b))
$$

and hence $\mathcal{F}(L(a, b)) \simeq 0$, as desired. 
2.5. Quotients and localizations of the category R-gmod. In this subsection we will recall the quotient category and the localizations of $R$-gmod introduced in $[11, \S 4.4$ - §4.5]. For details of the constructions, see [11, Appendix A and B].

Set $\mathcal{A}_{\beta}=R(\beta)$-gmod and set $\mathcal{A}=\bigoplus_{\beta \in Q_{J}^{+}} \mathcal{A}_{\beta}$. Then we have constructed a functor $\mathcal{F}=\bigoplus_{\beta \in Q_{J}^{+}} \mathcal{F}_{\beta}: \mathcal{A} \rightarrow \mathscr{C}_{J}=\mathscr{C}_{B_{n}^{(1)}}^{0} \subset \mathscr{C}_{B_{n}^{(1)}}$

Let $\mathcal{S}_{N}$ be the smallest Serre subcategory of $\mathcal{A}$ such that

(1) $\mathcal{S}_{N}$ contains $L(a, a+N)$ for any $a \in \mathbb{Z}$,

(2) $X \circ Y, Y \circ X \in \mathcal{S}_{N}$ for all $X \in \mathcal{A}$ and $Y \in \mathcal{S}_{N}$.

Note that $\mathcal{S}_{N}$ contains $L(a, b)$ if $b \geq a+N$.

Let us denote by $\mathcal{A} / \mathcal{S}_{N}$ the quotient category of $\mathcal{A}$ by $\mathcal{S}_{N}$ and denote by $\mathcal{Q}: \mathcal{A} \rightarrow$ $\mathcal{A} / \mathcal{S}_{N}$ the canonical functor. Since $\mathcal{F}$ sends $\mathcal{S}_{N}$ to 0 by Proposition 2.4.6, the functor $\mathcal{F}: \mathcal{A} \rightarrow \mathscr{C}_{J}$ factors through $\mathcal{Q}$, i.e., there exists a functor $\mathcal{F}^{\prime}: \mathcal{A} / \mathcal{S}_{N} \rightarrow \mathscr{C}_{J}$ unique up to an isomorphism such that the diagram below quasi-commutes.

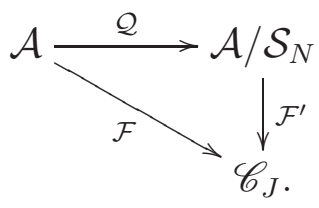

Note that $\mathcal{A}$ and $\mathcal{A} / \mathcal{S}_{N}$ are tensor categories with the convolution as tensor products. The module $R(0) \simeq \mathbf{k}$ is a unit object. Note also that $Q:=q R(0)$ is an invertible central object of $\mathcal{A} / \mathcal{S}_{N}$ and $X \mapsto Q \circ X \simeq X \circ Q$ coincides with the grading shift functor. Moreover, the functors $\mathcal{Q}, \mathcal{F}$ and $\mathcal{F}^{\prime}$ are tensor functors.

We will recall the localizations $\mathcal{T}_{N}^{\prime}$ and $\mathcal{T}_{N}$ of $\mathcal{A} / \mathcal{S}_{N}$ introduced in [11, §4.5] (where they are denoted by $T_{J}^{\prime}$ and $T_{J}$, respectively).

Definition 2.5.1. Let $S$ be the automorphism of $P_{J}:=\bigoplus_{a \in \mathbb{Z}} \mathbb{Z} \epsilon_{a}$ given by $S\left(\epsilon_{a}\right)=\epsilon_{a+N}$. We define the bilinear form $B$ on $P_{J}$ by

$$
B(x, y)=-\sum_{k>0}\left(S^{k} x, y\right) \quad \text { for } x, y \in P_{J} .
$$

Definition 2.5.2. We define the new tensor product $\star: \mathcal{A} / \mathcal{S}_{N} \times \mathcal{A} / \mathcal{S}_{N} \rightarrow \mathcal{A} / \mathcal{S}_{N}$ by

$$
X \star Y=q^{B(\alpha, \beta)} X \circ Y
$$

where $X \in\left(\mathcal{A} / \mathcal{S}_{N}\right)_{\alpha}$ and $Y \in\left(\mathcal{A} / \mathcal{S}_{N}\right)_{\beta}$.

Then $\mathcal{A} / \mathcal{S}_{N}$ is endowed with a new structure of tensor category by $\star$ as shown in [11, Appendix A.8].

Set

$$
L_{a}:=L(a, a+N-1) \quad \text { for } a \in \mathbb{Z}
$$


For $a, j \in \mathbb{Z}$, set

$$
f_{a, j}(z):=(-1)^{\delta_{j, a+N}} z^{-\delta(a \leq j<a+N-1)-\delta_{j, a+N}} \in \mathbf{k}\left[z^{ \pm 1}\right] .
$$

Theorem 2.5.3 ([11, Theorem 4.5.8]). The following statements hold.

(i) $L_{a}$ is a central object in $\mathcal{A} / \mathcal{S}_{N}$; i.e.,

(a) $f_{a, j}(z) R_{L_{a}, L(j)_{z}}$ induces an isomorphism in $\mathcal{A} / \mathcal{S}_{N}$

$$
R_{a}(X): L_{a} \star X \stackrel{\sim}{\longrightarrow} \star L_{a}
$$

functorial in $X \in \mathcal{A} / \mathcal{S}_{N}$

(b) the diagram

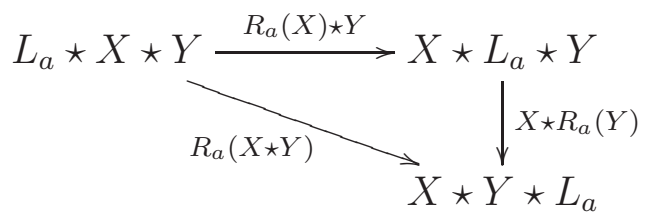

is commutative in $\mathcal{A} / \mathcal{S}_{N}$ for any $X, Y \in \mathcal{A} / \mathcal{S}_{N}$.

(ii) The isomorphism $R_{a}\left(L_{a}\right): L_{a} \star L_{a} \stackrel{\sim}{\longrightarrow} L_{a} \star L_{a}$ coincides with $\operatorname{id}_{L_{a} \star L_{a}}$ in $\mathcal{A} / \mathcal{S}_{N}$.

(iii) For $a, b \in \mathbb{Z}$, the isomorphisms

$$
R_{a}\left(L_{b}\right): L_{a} \star L_{b} \stackrel{\sim}{\longrightarrow} L_{b} \star L_{a} \text { and } R_{b}\left(L_{a}\right): L_{b} \star L_{a} \stackrel{\sim}{\longrightarrow} L_{a} \star L_{b}
$$

in $\mathcal{A} / \mathcal{S}_{N}$ are inverse to each other.

By the preceding theorem, $\left\{\left(L_{a}, R_{a}\right)\right\}_{a \in J}$ forms a commuting family of central objects in $\left(\mathcal{A} / \mathcal{S}_{N}, \star\right)$ (See [11, Appendix A. 4]). Following [11, Appendix A. 6], we localize $\left(\mathcal{A} / \mathcal{S}_{N}, \star\right)$ by this commuting family. Let us denote by $\mathcal{T}_{N}^{\prime}$ the resulting category $\left(\mathcal{A} / \mathcal{S}_{N}\right)\left[L_{a}^{\star-1} \mid a \in J\right]$. Let $\Upsilon: \mathcal{A} / \mathcal{S}_{N} \rightarrow \mathcal{T}_{N}^{\prime}$ be the projection functor. We denote by $\mathcal{T}_{N}$ the tensor category $\left(\mathcal{A} / \mathcal{S}_{N}\right)\left[L_{a} \simeq \mathbf{1} \mid a \in J\right]$ and by $\Xi: \mathcal{T}_{N}^{\prime} \rightarrow \mathcal{T}_{N}$ the canonical functor (see [11, Appendix A.7] and [11, Remark 4.5.9]). Thus we have a chain of tensor functors

$$
\mathcal{A} \stackrel{\mathcal{Q}}{\longrightarrow} \mathcal{A} / \mathcal{S}_{N} \stackrel{\Upsilon}{\longrightarrow} \mathcal{T}_{N}^{\prime}:=\left(\mathcal{A} / \mathcal{S}_{N}\right)\left[L_{a}^{\star-1} \mid a \in J\right] \stackrel{\Xi}{\longrightarrow} \mathcal{T}_{N}:=\left(\mathcal{A} / \mathcal{S}_{N}\right)\left[L_{a} \simeq 1 \mid a \in J\right] .
$$

The categories $\mathcal{T}_{N}$ and $\mathcal{T}_{N}^{\prime}$ are rigid tensor categories; i.e., every object has a right dual and a left dual ([11, Theorem 4.6.3]).

Let us denote by $\operatorname{Irr}\left(\mathcal{T}_{N}\right)$ the set of the isomorphism classes of simple objects in $\mathcal{T}_{N}$. Define an equivalence relation $\sim$ on $\operatorname{Irr}\left(\mathcal{T}_{N}\right)$ by $X \sim Y$ if and only if $X \simeq q^{c} Y$ in $\mathcal{T}_{N}$ for some integer $c$. We write $\operatorname{Irr}\left(\mathcal{T}_{N}\right)_{q=1}$ for $\operatorname{Irr}\left(\mathcal{T}_{N}\right) / \sim$.

Proposition 2.5.4 ([11, Proposition 4.7.3 (iii)]). The set $\operatorname{Irr}\left(\mathcal{T}_{N}\right)_{q=1}$ is isomorphic to the set of ordered multisegments

$$
\left(\left(a_{1}, b_{1}\right), \ldots,\left(a_{r}, b_{r}\right)\right)
$$

satisfying

$$
b_{k}-a_{k}+1<N \text { for any } 1 \leq k \leq r,
$$


by the correspondence

$$
\left(\left(a_{1}, b_{1}\right), \ldots,\left(a_{r}, b_{r}\right)\right) \mapsto(\Xi \circ \Upsilon \circ \mathcal{Q})\left(\operatorname{hd}\left(L\left(a_{1}, b_{1}\right) \circ \cdots \circ L\left(a_{r}, b_{r}\right)\right)\right) .
$$

2.6. Factoring through $\mathcal{T}_{N}$. Let us come back to the $B_{n}^{(1)}$-case. Recall that $N=2 n$. We have constructed the tensor functor $\mathcal{F}: \mathcal{A} \longrightarrow \mathscr{C}_{B_{n}^{(1)}}^{(1)}$. In this subsection, we will show that the functor $\mathcal{F}$ factors through the category $\mathcal{T}_{N}$ under a suitable choice of duality coefficient $\left\{P_{i, j}(u, v)\right\}_{i, j \in J}$.

First, we need the following lemma.

Lemma 2.6.1. Set $V(a):=\mathcal{F}(L(a))$ for $a \in J$. Recall that $V(a)=V\left(\varpi_{i_{a}}\right)_{X(a)}$ for some $i_{a} \in I_{0}$. For $a, b \in J$, set

$$
\begin{aligned}
& R_{a, b}^{\text {norm }}=\left(R_{V(a), V(b)_{z_{V}(b)}}^{\text {norm }} \otimes 1 \otimes \cdots \otimes 1\right) \circ\left(1 \otimes R_{V(a+1), V(b)}^{\text {norm }} \otimes 1 \otimes \cdots \otimes 1\right) \circ \cdots \\
& \quad \quad\left(1 \otimes \cdots \otimes 1 \otimes R_{V(a+k), V(b)_{z_{V}(b)}}^{\text {norm }} \otimes 1 \otimes \cdots \otimes 1\right) \circ \cdots \circ\left(1 \otimes \cdots \otimes 1 \otimes R_{V(a+N-1), V(b)_{z_{V}(b)}}^{\text {norm }}\right)
\end{aligned}
$$

and

$$
g_{a, b}(z):=\prod_{k=0}^{N-1} a_{V\left(\varpi_{i_{a+k}}\right), V\left(\varpi_{i_{b}}\right)}\left(X(a+k)^{-1} X(b)(1+z)\right)^{-1},
$$

where $z=z_{V(b)}-1$ and $a_{M, N}\left(z_{N}\right) \in \mathbf{k}\left[\left[z_{N}\right]\right]^{\times}$denotes the ratio of $R_{M, N_{z_{N}}}^{\text {univ }}$ and $R_{M, N_{z_{N}}}^{\text {norm }}$, i.e., $R_{M, N_{z_{N}}}^{\text {univ }}=a_{M, N}\left(z_{N}\right) R_{M, N_{z_{N}}}^{\text {norm }}$. Then the following diagram is commutative

$$
\begin{array}{cc}
(V(a) \otimes \cdots \otimes V(a+N-1)) \otimes V(b)_{z_{V(b)}} \stackrel{R_{a, b}^{\mathrm{norm}}}{\longrightarrow} V(b)_{z_{V(b)}} \otimes(V(a) \otimes \cdots \otimes V(a+N-1)) \\
\qquad \varphi \otimes V(b)_{z_{V(b)}} & \downarrow^{V(b) z_{V(b)} \otimes \varphi} \\
\mathbf{k} \otimes V(b)_{z_{V(b)}} & V(b)_{z_{V(b)}} \otimes \mathbf{k} \\
\sim \downarrow & \sim \downarrow \\
V(b)_{z_{V(b)}} & \sim V(b)_{z_{V(b)}}
\end{array}
$$

for any surjective homomorphism $\varphi: V(a) \otimes \cdots \otimes V(a+N-1) \rightarrow \mathbf{k}$.

Proof. Let

$$
\begin{aligned}
R^{\text {univ }}=\left(R_{V(a), V(b)_{z_{V}(b)}}^{\text {univ }} \otimes 1 \otimes \cdots \otimes 1\right) \circ( & 1 \otimes R_{\left.V(a+1), V(b)_{z_{V(b)}}^{\text {univ }} \otimes 1 \otimes \cdots \otimes 1\right)} \\
& \circ \cdots \circ\left(1 \otimes \cdots \otimes 1 \otimes R_{V(a+N-1), V(b)_{z_{V}(b)}}^{\text {univ }}\right) .
\end{aligned}
$$

If $R^{\text {norm }}$ and $g_{a, b}(z)$ in the diagram are replaced by $R^{\text {univ }}$ and the identity map respectively, then the square is commutative. Hence the lemma follows from $a_{M_{x}, N_{y}}(z)=$ $a_{M, N}\left(x^{-1} y z\right)$ for any simple $U_{q}^{\prime}(\mathfrak{g})$ modules $M, N$ and $x, y \in \mathbf{k}^{\times}$.

We temporarily fix a duality coefficient $\left\{P_{i, j}^{\mathrm{T}}(u, v)\right\}_{i, j \in J}$, e.g. as in (2.10), and denote the corresponding functor from $\mathcal{A} / \mathcal{S}_{N}$ to $\mathscr{C}_{B_{n}^{(1)}}^{0}$ by $\mathcal{F}^{\mathrm{T}}$. Recall $L_{a}=L(a, a+N-1)$ from (2.16) and $f_{a, b}(z)$ from (2.17). 
Proposition 2.6.2. Let

$$
h_{a, b}(z):=f_{a, b}(z) g_{a, b}(z) \prod_{k=a}^{a+N-1} P_{k, b}^{\mathrm{T}}(0, z)
$$

Then the following diagram is commutative

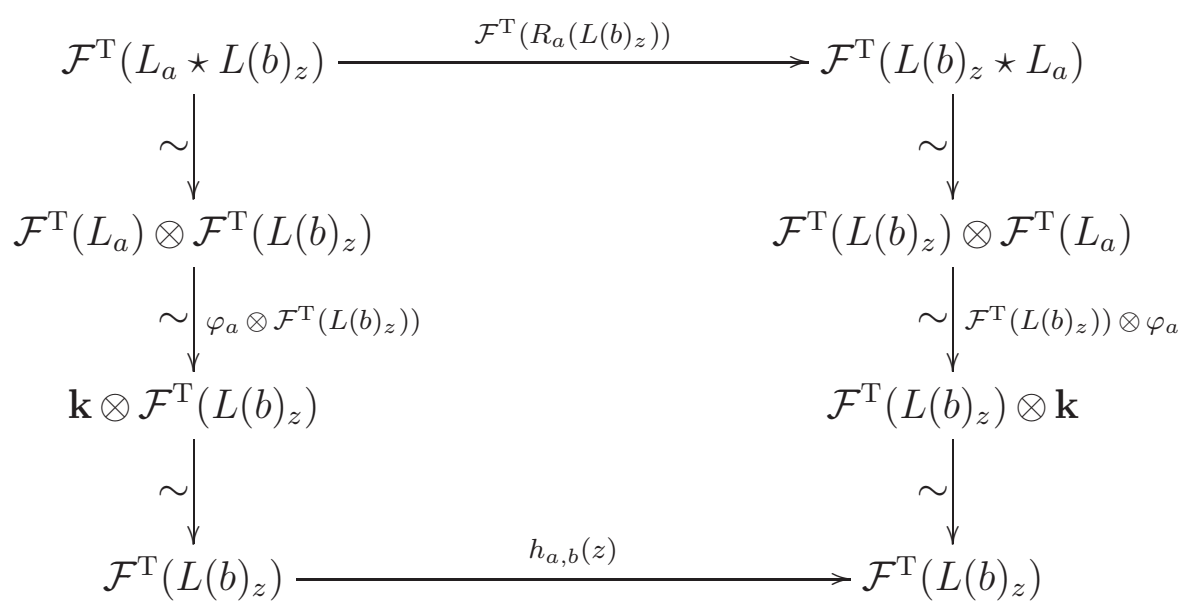

for any isomorphism $\varphi_{a}: \mathcal{F}^{\mathrm{T}}\left(L_{a}\right) \stackrel{\sim}{\sim} \mathbf{k}$.

Proof. Combining (1.22) with the above lemma, we have a commutative diagram

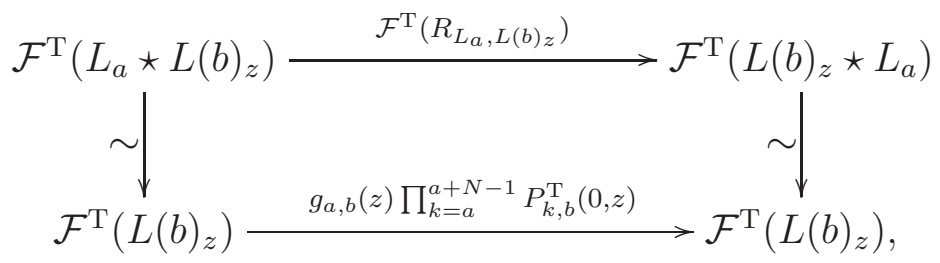

where the vertical isomorphisms are the compositions of the isomorphisms of the diagram in the proposition. Since $R_{a}\left(L(b)_{z}\right)=f_{a, b}(z) R_{L_{a}, L(b) z}$ by Theorem 2.5.3 (i) (a), we get the desired result.

Corollary 2.6.3. The function $h_{a, b}(z)$ has no poles and no zeros at $z=0$.

Proof. Since $R_{a}\left(L(b)_{z}\right)$ is an isomorphism, $\mathcal{F}^{\mathrm{T}}\left(R_{a}\left(L(b)_{z}\right)\right) \simeq h_{a, b}(z)$ is a well-defined isomorphism. Hence $h_{a, b}(z)$ has no pole and no zero at $z=0$. 
Proposition 2.6.4. The following diagram is commutative

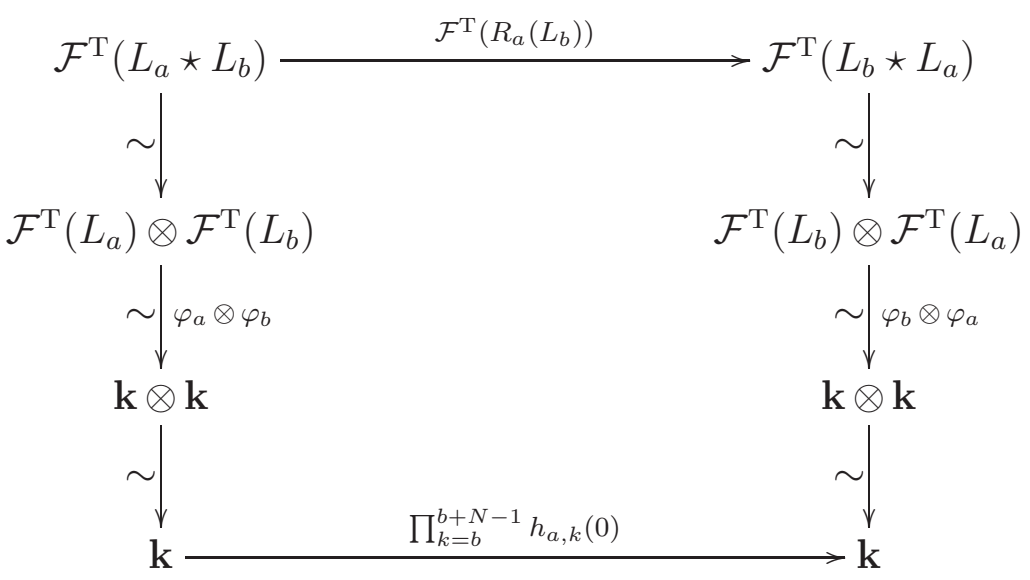

for any isomorphisms $\varphi_{a}: \mathcal{F}^{\mathrm{T}}\left(L_{a}\right) \stackrel{\sim}{\sim}$ and $\varphi_{b}: \mathcal{F}^{\mathrm{T}}\left(L_{b}\right) \stackrel{\sim}{\sim} \mathbf{k}$

Proof. Let $h$ be a composition of homomorphisms in $\mathcal{A} / \mathcal{S}_{N}$ as follows:

$$
\begin{aligned}
& h:=\left((L(b) \star \cdots \star L(b+N-2)) \star R_{a}(L(b+N-1))\right) \circ \cdots \\
& \circ\left((L(b) \star \cdots \star L(b+k-1)) \star R_{a}(L(b+k)) \star(L(b+k+1) \star \cdots \star L(b+N-1))\right) \circ \cdots \\
& \circ\left(R_{a}(L(b)) \star(L(b+1) \star \cdots \star L(b+N-1))\right) .
\end{aligned}
$$

Consider the following diagram

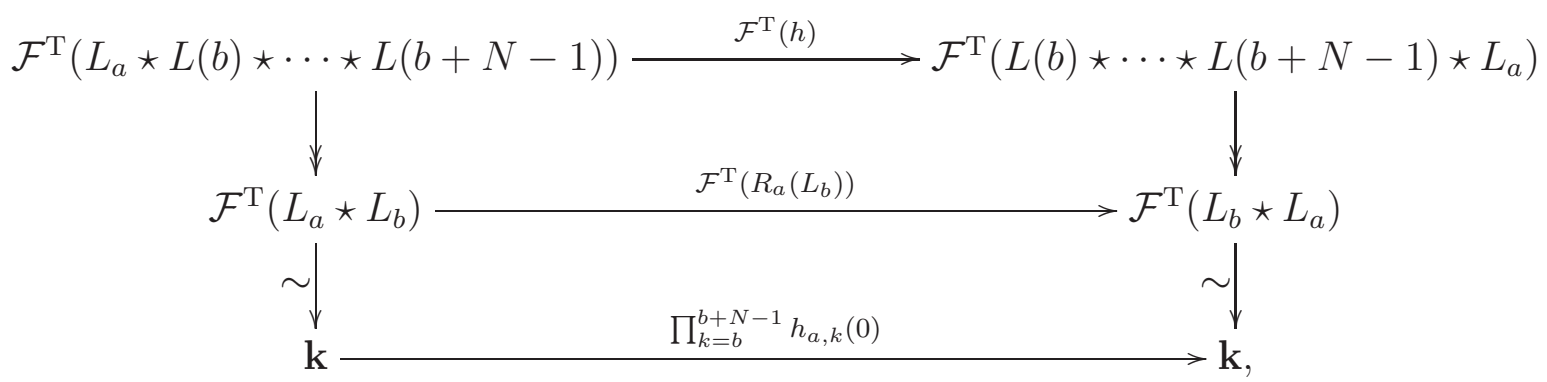

where the vertical isomorphisms are the compositions of the isomorphisms of the diagram in the proposition.

The upper square is commutative. By Proposition 2.6.2, the outer square is also commutative. Hence we get the commutativity of the lower square, as desired.

Corollary 2.6.5. Set $\lambda_{a, b}:=\prod_{k=b}^{b+N-1} h_{a, k}(0)$. Then we have

$$
\lambda_{a, a}=1 \quad(a \in \mathbb{Z}) \quad \text { and } \quad \lambda_{a, b} \lambda_{b, a}=1 \quad(a, b \in \mathbb{Z}) .
$$

Proof. By Theorem 2.5.3 (ii), we have $R_{a}\left(L_{a}\right)=\mathrm{id}_{L_{a} \star L_{a}}$ and hence $\mathcal{F}^{\mathrm{T}}\left(R_{a}\left(L_{a}\right)\right)$ is the identity map on $\mathbf{k}$. Hence $\lambda_{a, a}=1$ by the above proposition. Similarly, (iii) in Theorem 2.5.3 implies $\lambda_{a, b} \lambda_{b, a}=1$. 
Lemma 2.6.6. Let $\mathbf{k}$ be a field and let $N$ be a positive integer. Let $\left\{h_{a, b}\right\}_{a, b \in \mathbb{Z}}$ be $a$ family of elements in $\mathbf{k}^{\times}$. Set

$$
\lambda_{a, b}:=\prod_{k=b}^{b+N-1} h_{a, k} \quad(a, b \in \mathbb{Z}) .
$$

Then the system of equations in $\left\{c_{a, b}\right\}_{a, b \in \mathbb{Z}}$

$$
\left\{\begin{array}{l}
\prod_{k=a}^{a+N-1} c_{k, b}=h_{a, b} \quad(a, b \in \mathbb{Z}) \\
c_{a, a}=1 \quad(a \in \mathbb{Z}) \quad \text { and } \quad c_{a, b} c_{b, a}=1 \quad(a, b \in \mathbb{Z})
\end{array}\right.
$$

has a solution if and only if

$$
\lambda_{a, a}=1 \quad(a \in \mathbb{Z}) \quad \text { and } \quad \lambda_{a, b} \lambda_{b, a}=1 \quad(a, b \in \mathbb{Z}),
$$

Proof. The "only if" part is straightforward because $\lambda_{a, b}=\prod_{k=a}^{a+N-1} \prod_{j=b}^{b+N-1} c_{k, j}$.

Assume that (2.21) holds. By the construction, we have

$$
\frac{\lambda_{a, b+1}}{\lambda_{a, b}}=\frac{h_{a, b+N}}{h_{a, b}} \quad \text { for any } a, b \in \mathbb{Z} \text {. }
$$

First, we take an arbitrary $\left\{c_{a, b}\right\}_{0 \leq a, b \leq N-2}$ satisfying $c_{a, a}=1, c_{a, b} c_{b, a}=1$.

Next, we extend it to $\left\{c_{a, b}\right\}_{0 \leq a, b \leq N-1}$ by

$$
\begin{aligned}
& c_{N-1, b}:=h_{0, b} \prod_{k=0}^{N-2} c_{k, b}^{-1} \quad(0 \leq b \leq N-2), \\
& c_{a, N-1}:=\left(c_{N-1, a}\right)^{-1} \quad(0 \leq a \leq N-2), \\
& c_{N-1, N-1}=1 .
\end{aligned}
$$

Then we define $c_{a, b}$ for all $a, b \in \mathbb{Z}$ by extending them under the conditions

$$
\begin{aligned}
c_{a+N, b}:=\frac{h_{a+1, b}}{h_{a, b}} c_{a, b} \quad \text { and } \\
c_{a, b+N}:=\frac{h_{b, a}}{h_{b+1, a}} c_{a, b} \quad \text { for all } a, b \in \mathbb{Z} .
\end{aligned}
$$

Note that such an extension is possible because $\varphi_{a, b}:=\frac{h_{a+1, b}}{h_{a, b}}$ and $\psi_{a, b}:=\frac{h_{b, a}}{h_{b+1, a}}$ satisfy the cocycle condition

$$
\varphi_{a, b+N} \psi_{a, b}=\psi_{a+N, b} \varphi_{a, b}
$$

as seen in

$$
\begin{aligned}
\frac{\varphi_{a, b+N} \psi_{a, b}}{\psi_{a+N, b} \varphi_{a, b}} & =\frac{h_{a+1, b+N}}{h_{a, b+N}} \frac{h_{b, a}}{h_{b+1, a}} \frac{h_{b+1, a+N}}{h_{b, a+N}} \frac{h_{a, b}}{h_{a+1, b}}=\frac{h_{a+1, b+N}}{h_{a+1, b}} \frac{h_{a, b}}{h_{a, b+N}} \frac{h_{b, a}}{h_{b, a+N}} \frac{h_{b+1, a+N}}{h_{b+1, a}} \\
& =\frac{\lambda_{a+1, b+1}}{\lambda_{a+1, b}} \frac{\lambda_{a, b}}{h_{a, b+1}} \frac{\lambda_{b, a}}{\lambda_{b, a+1}} \frac{\lambda_{b+1, a+1}}{\lambda_{b+1, a}}=1 .
\end{aligned}
$$

Here the third equality follows from (2.22). By the construction, we can easily verify

$$
c_{a, a}=1 \text { for } 0 \leq a \leq N-1 \text { and } c_{a, b} c_{b, a}=1 \text { for } 0 \leq a, b \leq N-1 \text {. }
$$


Then we have

$$
c_{a, b+N} c_{b+N, a}=\frac{h_{b, a}}{h_{b+1, a}} \frac{h_{b+1, a}}{h_{b, a}} c_{a, b} c_{b, a}=c_{a, b} c_{b, a},
$$

and similarly

$$
c_{a+N, b} c_{b, a+N}=c_{a, b} c_{b, a}
$$

for $a, b \in \mathbb{Z}$. It follows from (2.25) that

$$
c_{a, b} c_{b, a}=1 \quad \text { for all } a, b \in \mathbb{Z} .
$$

By (2.22), we get

$$
\begin{aligned}
c_{a+N, a+N} & =\frac{h_{a+1, a+N}}{h_{a, a+N}} c_{a, a+N}=\frac{h_{a+1, a+N}}{h_{a, a+N}} \frac{h_{a, a}}{h_{a+1, a}} c_{a, a} \\
& =\frac{h_{a+1, a+N}}{h_{a+1, a}} \frac{h_{a, a}}{h_{a, a+N}} c_{a, a}=\frac{\lambda_{a+1, a+1}}{\lambda_{a+1, a}} \frac{\lambda_{a, a}}{\lambda_{a, a+1}} c_{a, a}=c_{a, a}
\end{aligned}
$$

for all $a \in \mathbb{Z}$. Thus (2.25) implies $c_{a, a}=1$ for all $a \in \mathbb{Z}$.

Note that by the definition we have

$$
\prod_{k=0}^{N-1} c_{k, b}=h_{0, b} \quad(0 \leq b \leq N-2) .
$$

When $b=N-1$, we have

$$
\prod_{k=0}^{N-1} c_{k, N-1}=\prod_{k=0}^{N-1} c_{N-1, k}^{-1}=\prod_{k=0}^{N-2} c_{N-1, k}^{-1}=\prod_{k=0}^{N-2}\left(h_{0, k}^{-1} \prod_{j=0}^{N-2} c_{j, k}\right) .
$$

Since $\prod_{0 \leq j, k \leq N-2} c_{j, k}=1$, we have

$$
\prod_{k=0}^{N-1} c_{k, N-1}=\prod_{k=0}^{N-2} h_{0, k}^{-1}=h_{0, N-1} \lambda_{0,0}^{-1}=h_{0, N-1} .
$$

Hence we have showed that

$$
\prod_{k=a}^{a+N-1} c_{k, b}=h_{a, b}
$$

for $a=0$ and $0 \leq b \leq N-1$. Then by (2.23), it holds for all $a \in \mathbb{Z}$ and $0 \leq b \leq N-1$. Now by (2.24), it holds for all $a \in \mathbb{Z}$ and $b \in \mathbb{Z}$, as desired.

Lemma 2.6.7. Let $\left\{h_{a, b}(z)\right\}_{a, b \in \mathbb{Z}}$ be a family of elements in $\mathbf{k}[[z]]$. Assume that

$$
\lambda_{a, b}:=\prod_{k=b}^{b+N-1} h_{a, k}(0) \quad(a, b \in \mathbb{Z})
$$

satisfies that

$$
\lambda_{a, a}=1 \quad(a \in \mathbb{Z}) \quad \text { and } \quad \lambda_{a, b} \lambda_{b, a}=1 \quad(a, b \in \mathbb{Z}) .
$$


Then there exists a family $\left\{c_{a, b}(u, v)\right\}_{a, b \in \mathbb{Z}}$ of elements in $\mathbf{k}[[u, v]]$ satisfying

$$
\begin{aligned}
& c_{a, a}(u, v)=1 \quad(a \in \mathbb{Z}), \quad c_{a, b}(u, v) c_{b, a}(v, u)=1 \quad(a, b \in \mathbb{Z}), \\
& \text { and } \quad h_{a, b}(z)=\prod_{k=a}^{a+N-1} c_{k, b}(0, z) \quad(a, b \in \mathbb{Z}) .
\end{aligned}
$$

Proof. By Lemma 2.6.6, there exists a family $\left\{c_{a, b}\right\}_{a, b \in \mathbb{Z}}$ of elements in $\mathbf{k}^{\times}$satisfying

$$
\begin{aligned}
& c_{a, a}=1 \quad(a \in \mathbb{Z}), \quad c_{a, b} c_{b, a}=1 \quad(a, b \in \mathbb{Z}), \\
& \text { and } h_{a, b}(0)=\prod_{k=a}^{a+N-1} c_{k, b} \quad(a, b \in \mathbb{Z}) .
\end{aligned}
$$

Set $\varphi_{a, a}(v)=1 \in \mathbf{k}[[v]]$ for $a \in \mathbb{Z}$. For $a, b \in \mathbb{Z}$ such that $a<b<a+N-1$, we choose $\varphi_{a, b}(v) \in \mathbf{k}[[v]]$ satisfying $\varphi_{a, b}(0)=c_{a, b}$. Then $\varphi_{a, b}(0)=c_{a, b}$ for $a, b \in \mathbb{Z}$ with $a \leq b<a+N-1$. We extend them to all $a, b \in \mathbb{Z}$ inductively

$$
\varphi_{a, b}(v):= \begin{cases}\frac{h_{a-N+1, b}(v)}{\prod_{k=a-N+1}^{a-1} \varphi_{k, b}(v)}, & \text { for } b<a, \\ \frac{h_{a, b}(v)}{\prod_{a+1}^{k=a+N-1} \varphi_{k, b}(v)}, & \text { for } b \geq a+N-1 .\end{cases}
$$

Then we have

$$
\varphi_{a, b}(0)=c_{a, b} \quad \text { for } a, b \in \mathbb{Z}, \quad \text { and } \prod_{k=a}^{a+N-1} \varphi_{k, b}(v)=h_{a, b}(v) \quad \text { for } a, b \in \mathbb{Z} .
$$

Define

$$
c_{a, b}(u, v):=\frac{\varphi_{a, b}(v)}{\varphi_{b, a}(u)} c_{b, a} \in \mathbf{k}[[u, v]] \quad \text { for } a, b \in \mathbb{Z} .
$$

Then $c_{a, a}(u, v)=1$ holds trivially, and we have

$$
c_{a, b}(u, v) c_{b, a}(v, u)=\frac{\varphi_{a, b}(v)}{\varphi_{b, a}(u)} \frac{\varphi_{b, a}(u)}{\varphi_{a, b}(v)} c_{b, a} c_{a, b}=1 .
$$

We also have

$$
c_{a, b}(0, z)=\frac{\varphi_{a, b}(z)}{\varphi_{b, a}(0)} c_{b, a}=\varphi_{a, b}(z)
$$

and hence

$$
h_{a, b}(z)=\prod_{k=a}^{a+N-1} \varphi_{k, b}(z)=\prod_{k=a}^{a+N-1} c_{k, b}(0, z) \quad \text { for } a, b \in \mathbb{Z}
$$

as desired.

Let $\left\{c_{a, b}(u, v)\right\}_{a, b \in \mathbb{Z}}$ be a family obtained by applying Lemma 2.6.7 to the family $\left\{h_{a, b}(z)\right\}_{a, b \in \mathbb{Z}}$ in (2.6.2). Note that Corollary 2.6.5 asserts that the condition for $\left\{h_{a, b}(z)\right\}_{a, b \in \mathbb{Z}}$ in Lemma 2.6.7 holds. 
We now give a new duality coefficient by

$$
P_{a, b}^{\text {new }}(u, v):=c_{a, b}(u, v)^{-1} P_{a, b}^{\mathrm{T}}(u, v)=c_{b, a}(v, u) P_{a, b}^{\mathrm{T}}(u, v),
$$

and denote the corresponding functor from $\mathcal{A} / \mathcal{S}_{N}$ to $\mathscr{C}_{B_{n}^{(1)}}$ by $\mathcal{F}^{\prime}$. Note that the new duality coefficient $\left\{P_{a, b}^{\text {new }}(u, v)\right\}_{a, b \in \mathbb{Z}}$ also satisfies the conditions in (1.17) including the equality

$$
Q_{a, b}(u, v)=\delta(a \neq b) P_{a, b}^{\text {new }}(u, v) P_{b, a}^{\text {new }}(v, u)
$$

since $c_{a, b}(u, v) c_{b, a}(v, u)=1$.

Theorem 2.6.8. Let $a \in J$ and $M \in \mathcal{A} / \mathcal{S}_{N}$. The the diagram

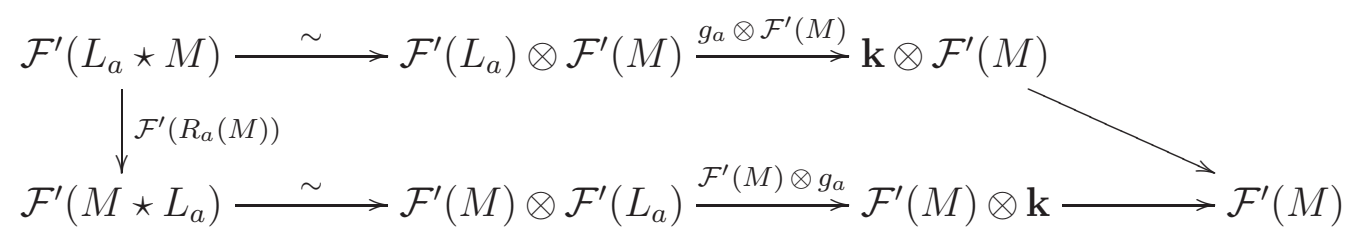

is commutative for any isomorphism $g_{a}: \mathcal{F}^{\prime}\left(L_{a}\right) \stackrel{\sim}{\sim}$.

Proof. It is enough to show the commutativity of the diagram in the cases $M=$ $L(b)_{z}$ for $b \in J$. By the same reasoning in Proposition 2.6.2, we need to show $f_{a, b}(z) g_{a, b}(z) \prod_{k=a}^{a+N-1} P_{k, b}^{\text {new }}(0, z)=1$. This follows from

$$
f_{a, b}(z) g_{a, b}(z) \prod_{k=a}^{a+N-1} P_{k, b}^{\mathrm{new}}(0, z)=h_{a, b}(z) \prod_{k=a}^{a+N-1} c_{k, b}(0, z)^{-1}=1,
$$

where the last equality is a consequence of Lemma 2.6.7.

Then [11, Proposition A.7.3] and [11, Proposition A.7.2] imply

Theorem 2.6.9. There exists an exact tensor functor $\widetilde{\mathcal{F}}: \mathcal{T}_{N} \rightarrow \mathscr{C}_{B_{n}^{(1)}}^{0}$ such that the following diagram quasi-commutes:

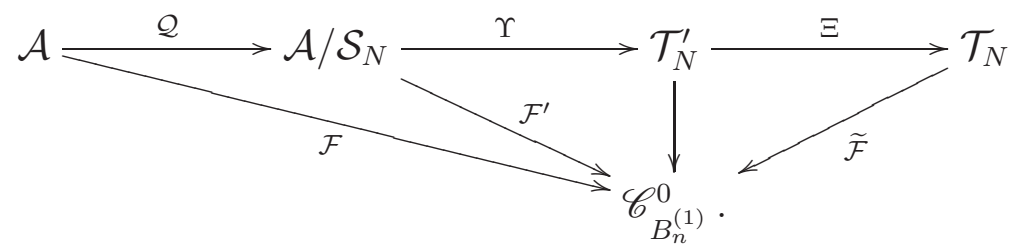

Hence $\widetilde{\mathcal{F}}$ induces a surjective ring homomorphism $\phi_{\widetilde{\mathcal{F}}}: K\left(\mathcal{T}_{N}\right)_{q=1} \rightarrow K\left(\mathscr{C}_{B_{n}^{(1)}}^{0}\right)$, where $K\left(\mathcal{T}_{N}\right)_{q=1}:=K\left(\mathcal{T}_{N}\right) /(q-1) K\left(\mathcal{T}_{N}\right)$

Corollary 2.6.10. The functor $\widetilde{\mathcal{F}}$ sends a non-zero object in $\mathcal{T}_{N}$ to a non-zero module in $\mathscr{C}_{B_{n}^{(1)}}^{(1)}$. In particular, it sends a simple to a simple.

Proof. Let $M$ be a non-zero object in $\mathcal{T}_{N}$. Since the category $\mathcal{T}_{N}$ is rigid, there exists $M^{*} \in \mathcal{T}_{N}$ such that there is an epimorphism $M^{*} \star M \rightarrow \mathbf{k}$. Since $\widetilde{\mathcal{F}}$ is exact, we have a surjective homomorphism $\widetilde{\mathcal{F}}\left(M^{*}\right) \otimes \widetilde{\mathcal{F}}(M) \rightarrow \mathbf{k}$. In particular, $\widetilde{\mathcal{F}}(M)$ is non-zero. 
In the rest of this subsection, we will show that $\phi_{\widetilde{\mathcal{F}}}$ is an isomorphism and induces a bijection between the classes of simple objects.

Lemma 2.6.11. Let $S$ and $S^{\prime}$ be simple modules in $\mathcal{A}$ with $S \simeq \operatorname{hd}\left(L\left(a_{1}, b_{1}\right) \circ\right.$ $\left.\cdots \circ L\left(a_{r}, b_{r}\right)\right)$ and $S^{\prime} \simeq \operatorname{hd}\left(L\left(a_{1}^{\prime}, b_{1}^{\prime}\right) \circ \ldots \circ L\left(a_{t}^{\prime}, b_{t}^{\prime}\right)\right)$ where $\left(\left(a_{1}, b_{1}\right), \ldots,\left(a_{r}, b_{r}\right)\right)$ and $\left(\left(a_{1}^{\prime}, b_{1}^{\prime}\right), \ldots,\left(a_{t}^{\prime}, b_{t}^{\prime}\right)\right)$ are ordered multisegments such that $b_{i}-a_{i}+1 \leq N(1 \leq i \leq r)$ and $b_{j}^{\prime}-a_{j}^{\prime}+1 \leq N(1 \leq j \leq t)$. Assume that $a_{1}=a_{1}^{\prime}$ and $\mathcal{F}(S) \simeq \mathcal{F}\left(S^{\prime}\right)$. Then we have

$$
\mathcal{F}\left(S_{0}\right) \simeq \mathcal{F}\left(S_{0}^{\prime}\right) \not 00
$$

where $S_{0}=\operatorname{hd}\left(L\left(a_{1}+1, b_{1}\right) \circ \cdots \circ L\left(a_{r}, b_{r}\right)\right)$ and $S_{0}^{\prime}=\operatorname{hd}\left(L\left(a_{1}^{\prime}+1, b_{1}^{\prime}\right) \circ \cdots \circ L\left(a_{t}^{\prime}, b_{t}^{\prime}\right)\right)$.

Proof. By the condition on the lengths of segments, $S$ and $S^{\prime}$ are simple as objects of $\mathcal{T}_{N}$. Hence $\mathcal{F}(S) \simeq \mathcal{F}\left(S^{\prime}\right)$ is a simple module by Corollary 2.6.10. By [25, Theorem 3.2 , Corollary 6.1], we have

$$
S \simeq L\left(a_{1}\right) \nabla S_{0} \text { and } S^{\prime} \simeq L\left(a_{1}\right) \nabla S_{0}^{\prime},
$$

which implies that there exists an epimorphism $\mathcal{F}\left(L\left(a_{1}\right)\right) \otimes \mathcal{F}\left(S_{0}\right) \rightarrow \mathcal{F}(S)$. Hence $\mathcal{F}\left(S_{0}\right)$ is non-zero and it is a simple module. Since $\mathcal{F}(S)$ is a simple module, we have $\mathcal{F}\left(L\left(a_{1}\right)\right) \nabla \mathcal{F}\left(S_{0}\right) \simeq \mathcal{F}(S)$ and similarly $\mathcal{F}\left(L\left(a_{1}\right)\right) \nabla \mathcal{F}\left(S_{0}^{\prime}\right) \simeq \mathcal{F}\left(S^{\prime}\right)$.

They imply that $\mathcal{F}\left(L\left(a_{1}\right)\right) \nabla \mathcal{F}\left(S_{0}\right) \simeq \mathcal{F}\left(L\left(a_{1}\right)\right) \nabla \mathcal{F}\left(S_{0}^{\prime}\right)$.

By [13, Corollary 3.14], we have $\mathcal{F}\left(S_{0}\right) \simeq \mathcal{F}\left(S_{0}^{\prime}\right)$, as desired.

Proposition 2.6.12. Let $S$ and $S^{\prime}$ be simple objects in $\mathcal{T}_{N}$ with

$$
\widetilde{\mathcal{F}}(S) \simeq \widetilde{\mathcal{F}}\left(S^{\prime}\right)
$$

then we have $S \simeq S^{\prime}$ in $\mathcal{T}_{N}$ up to a grading shift.

Proof. Let

$$
\left(\left(a_{1}, b_{1}\right), \ldots,\left(a_{r}, b_{r}\right)\right) \text { and }\left(\left(a_{1}^{\prime}, b_{1}^{\prime}\right), \ldots,\left(a_{t}^{\prime}, b_{t}^{\prime}\right)\right)
$$

be the multisegments associated with $S$ and $S^{\prime}$, respectively. Then we have $b_{i}-a_{i}+1<$ $N(1 \leq i \leq r)$ and $b_{j}^{\prime}-a_{j}^{\prime}+1<N(1 \leq j \leq t)$. We shall show that if $\widetilde{\mathcal{F}}(S) \simeq \widetilde{\mathcal{F}}\left(S^{\prime}\right)$, then we have $a_{1}=a_{1}^{\prime}$. Once we show it, then the proposition follows from the above lemma by induction on $\sum\left(b_{i}-a_{i}+1\right)+\sum\left(b_{j}^{\prime}-a_{j}^{\prime}+1\right)$.

Assume that $a_{1}>a_{1}^{\prime}$. Then the multisegment $\left(\left(a_{1}, a_{1}+N-1\right),\left(a_{1}^{\prime}, b_{1}^{\prime}\right), \ldots,\left(a_{t}^{\prime}, b_{t}^{\prime}\right)\right)$ is ordered. Hence we have

$$
\begin{aligned}
& \widetilde{\mathcal{F}}\left(\operatorname{hd}\left(L\left(a_{1}, a_{1}+N-1\right) \circ L\left(a_{1}^{\prime}, b_{1}^{\prime}\right) \circ \cdots \circ L\left(a_{t}^{\prime}, b_{t}^{\prime}\right)\right)\right) \\
& \quad \simeq \widetilde{\mathcal{F}}\left(S^{\prime}\right) \simeq \widetilde{\mathcal{F}}(S) \simeq \widetilde{\mathcal{F}}\left(\operatorname{hd}\left(L\left(a_{1}, b_{1}\right) \circ \cdots \circ L\left(a_{r}, b_{r}\right)\right)\right) .
\end{aligned}
$$

Applying Lemma 2.6.11 $\left(b_{1}-a_{1}+1\right)$ times, we have

$$
\begin{aligned}
\widetilde{\mathcal{F}}(\operatorname{hd} & \left.\left(L\left(b_{1}+1, a_{1}+N-1\right) \circ L\left(a_{1}^{\prime}, b_{1}^{\prime}\right) \circ \cdots \circ L\left(a_{t}^{\prime}, b_{t}^{\prime}\right)\right)\right) \\
& \simeq \widetilde{\mathcal{F}}\left(\operatorname{hd}\left(L\left(a_{2}, b_{2}\right) \circ \cdots \circ L\left(a_{r}, b_{r}\right)\right)\right) .
\end{aligned}
$$

Note that $b_{1}+1>a_{1} \geq a_{2}$. Thus we have

$$
\begin{aligned}
& \widetilde{\mathcal{F}}\left(\operatorname{hd}\left(L\left(b_{1}+1, a_{1}+N-1\right) \circ L\left(a_{1}^{\prime}, b_{1}^{\prime}\right) \circ \cdots \circ L\left(a_{t}^{\prime}, b_{t}^{\prime}\right)\right)\right) \\
& \quad \simeq \widetilde{\mathcal{F}}\left(\operatorname{hd}\left(L\left(b_{1}+1, b_{1}+N\right) \circ L\left(a_{2}, b_{2}\right) \circ \cdots \circ L\left(a_{r}, b_{r}\right)\right)\right) .
\end{aligned}
$$


Now we apply Lemma 2.6.11 again in order to obtain

$$
\begin{aligned}
\widetilde{\mathcal{F}}(\operatorname{hd} & \left.\left(L\left(a_{1}^{\prime}, b_{1}^{\prime}\right) \circ \cdots \circ L\left(a_{t}^{\prime}, b_{t}^{\prime}\right)\right)\right) \\
& \simeq \widetilde{\mathcal{F}}\left(\operatorname{hd}\left(L\left(a_{1}+N, b_{1}+N\right) \circ L\left(a_{2}, b_{2}\right) \circ \cdots \circ L\left(a_{r}, b_{r}\right)\right)\right) .
\end{aligned}
$$

Note that $a_{1}+N>a_{1}>a_{1}^{\prime}$. Hence, repeating the above procedure, we get

$$
\begin{aligned}
& \tilde{\mathcal{F}}(\operatorname{hd}\left.\left(L\left(a_{1}^{\prime}, b_{1}^{\prime}\right) \circ \cdots \circ L\left(a_{t}^{\prime}, b_{t}^{\prime}\right)\right)\right) \\
& \quad \simeq \widetilde{\mathcal{F}}\left(\operatorname{hd}\left(L\left(a_{1}+k N, b_{1}+k N\right) \circ L\left(a_{2}, b_{2}\right) \circ \cdots \circ L\left(a_{r}, b_{r}\right)\right)\right)
\end{aligned}
$$

for all $k \in \mathbb{Z}_{\geq 0}$.

Note that if $k$ is sufficiently large, then we have

$$
\begin{aligned}
& \operatorname{hd}\left(L\left(a_{1}+k N, b_{1}+k N\right) \circ L\left(a_{2}, b_{2}\right) \circ \cdots \circ L\left(a_{r}, b_{r}\right)\right) \\
& \quad \simeq L\left(a_{1}+k N, b_{1}+k N\right) \circ \operatorname{hd}\left(L\left(a_{2}, b_{2}\right) \circ \cdots \circ L\left(a_{r}, b_{r}\right)\right) .
\end{aligned}
$$

Hence it follows that

$$
\widetilde{\mathcal{F}}\left(S^{\prime}\right) \simeq \widetilde{\mathcal{F}}\left(L\left(a_{1}+k N, b_{1}+k N\right)\right) \otimes \widetilde{\mathcal{F}}\left(\operatorname{hd}\left(L\left(a_{2}, b_{2}\right) \circ \cdots \circ L\left(a_{r}, b_{r}\right)\right)\right)
$$

for all sufficiently large $k$. Hence the class of $\widetilde{\mathcal{F}}\left(\operatorname{hd}\left(L\left(a_{2}, b_{2}\right) \circ \cdots \circ L\left(a_{r}, b_{r}\right)\right)\right)$ in the Grothendieck ring $K\left(\mathscr{C}_{B_{n}^{(1)}}\right)$ is a zero-divisor. It contradicts the fact that $K\left(\mathscr{C}_{B_{n}^{(1)}}\right)$ is a polynomial ring generated by the classes of fundamental representations $([6])$.

Let us denote by $\operatorname{Irr}\left(\mathscr{C}_{B_{n}^{(1)}}^{0}\right)$ the set of isomorphism classes of simple objects in $\mathscr{C}_{B_{n}^{(1)}}^{0}$. The following is our main theorem.

Theorem 2.6.13. The functor $\widetilde{\mathcal{F}}: \mathcal{T}_{N} \rightarrow \mathscr{C}_{B_{n}^{(1)}}^{0}$ induces a ring isomorphism

$$
\phi_{\widetilde{\mathcal{F}}}: K\left(\mathcal{T}_{N}\right)_{q=1} \stackrel{\sim}{\longrightarrow} K\left(\mathscr{C}_{B_{n}^{(1)}}^{0}\right)
$$

and a bijection between $\mathcal{I} r r\left(\mathcal{T}_{N}\right)_{q=1}$ and $\mathcal{I} r r\left(\mathscr{C}_{B_{n}^{(1)}}^{0}\right)$.

Proof. We know that $\operatorname{Irr}\left(\mathcal{T}_{N}\right)_{q=1}$ is a basis of $K\left(\mathcal{T}_{N}\right)_{q=1}$ and $\operatorname{Irr}\left(\mathscr{C}_{B_{n}^{(1)}}^{0}\right)$ is a basis of $K\left(\mathscr{C}_{B_{n}^{(1)}}^{0}\right)$. By Proposition 2.6.12, the ring homomorphism $\phi_{\widetilde{\mathcal{F}}}$ sends $\mathcal{I} r r\left(\mathcal{T}_{N}\right)_{q=1}$ to $\operatorname{Irr}\left(\mathscr{C}_{B_{n}^{(1)}}^{0}\right)$ injectively. On the other hand, $\phi_{\widetilde{\mathcal{F}}}$ is surjective. Hence we conclude that $\phi_{\widetilde{\mathcal{F}}}$ is an isomorphism and induces a bijection between $\operatorname{Irr}\left(\mathcal{T}_{N}\right)_{q=1}$ and $\operatorname{Irr}\left(\mathscr{C}_{B_{n}^{(1)}}^{0}\right)$.

3. Relation between quantum affine algebras of type A And type B 3.1. Isomorphisms between Grothendieck rings. Recall from [11, 14] that for each $t=1,2$, there is a functor $\widetilde{\mathcal{F}}^{(t)}: \mathcal{T}_{N} \longrightarrow \mathscr{C}_{A_{N-1}^{(t)}}^{(t)}$ which induces a ring isomorphism

$$
\phi_{\widetilde{\mathcal{F}}(t)}: K\left(\mathcal{T}_{N}\right)_{q=1} \stackrel{\sim}{\longrightarrow} K\left(\mathscr{C}_{A_{N-1}^{(t)}}^{0}\right) .
$$

These ring isomorphisms $\phi_{\widetilde{\mathcal{F}}(t)}(t=1,2)$ are also bijective on the sets of classes of simple objects.

By setting

$$
\phi_{t}:=\phi_{\widetilde{\mathcal{F}}} \circ \phi_{\widetilde{\mathcal{F}}(t)}^{-1} \quad(t=1,2)
$$


we have

Theorem 3.1.1. There exist ring isomorphisms

$$
\phi_{t}: K\left(\mathscr{C}_{A_{N-1}^{(t)}}^{0}\right) \stackrel{\sim}{\longrightarrow} K\left(\mathscr{C}_{B_{n}^{(1)}}^{0}\right)
$$

which induce bijections between the sets of isomorphism classes of simple objects.

3.2. Images of modules associated with segments. In this subsection, we take

$$
\begin{aligned}
& \mathscr{S}_{0}\left(A_{2 n-1}^{(1)}\right):=\left\{\left(i,(-q)^{p}\right) \in\{1, \ldots, 2 n-1\} \times \mathbf{k}^{\times} ; p \equiv i+1 \bmod 2\right\}, \quad \text { and } \\
& \mathscr{S}_{0}\left(A_{2 n-1}^{(2)}\right):=\left\{\left(i, \pm(-q)^{p}\right) \in\{1, \ldots, n\} \times \mathbf{k}^{\times} ; i \in\{1, \ldots, n\}, p \equiv i+1 \bmod 2\right\} .
\end{aligned}
$$

The functors $\widetilde{\mathcal{F}}^{(t)}(t=1,2)$ send the simple module associated with a segment to a fundamental representation. More precisely, we have

$$
\phi_{\widetilde{\mathcal{F}}^{(1)}}([L(a, b)])=\left[V\left(\varpi_{b-a+1}\right)_{(-q)^{a+b}}\right]
$$

for $a, b \in \mathbb{Z}$, and

$$
\left(\phi_{\widetilde{\mathcal{F}}(2)} \circ \phi_{\widetilde{\mathcal{F}}(1)}^{-1}\right)\left(\left[V\left(\varpi_{i}\right)_{x}\right]\right)= \begin{cases}{\left[V\left(\varpi_{i}\right)_{x}\right]} & \text { for } 1 \leq i \leq n \\ {\left[V\left(\varpi_{N-i}\right)_{-x}\right]} & \text { for } n+1 \leq i \leq 2 n-1 .\end{cases}
$$

for every $(i, x) \in \mathscr{S}_{0}\left(A_{2 n-1}^{(1)}\right)$.

Hence the homomorphisms $\phi_{t}(t=1,2)$ send a fundamental representation to the image of the simple module associated with a segment under $\widetilde{\mathcal{F}}$.

Recall that $N=2 n$ and

$$
\begin{aligned}
& \widetilde{\mathcal{F}}(L(a, a+N-1)) \simeq \mathbf{k} \text { for all } a \in \mathbb{Z}, \\
& \widetilde{\mathcal{F}}(L(a+N, b+N)) \simeq \widetilde{\mathcal{F}}(L(a, b))_{q^{2 N-2}} \text { for all } a, b \in \mathbb{Z} .
\end{aligned}
$$

Hence the following proposition determines $\widetilde{\mathcal{F}}(L(a, b))$ for all $a, b \in \mathbb{Z}$.

Proposition 3.2.1. Let $0 \leq a \leq N-1$ and $0 \leq b-a \leq N-2$. Then we have

(i) $\widetilde{\mathcal{F}}(L(0, b)) \simeq V\left(\varpi_{n}\right)_{q^{2 b}}$ for $0 \leq b \leq N-2$.

(ii) $\widetilde{\mathcal{F}}(L(a, N-1)) \simeq V\left(\varpi_{n}\right)_{q^{2 a+N-3}} \quad$ for $1 \leq a \leq N-1$.

(iii) $\widetilde{\mathcal{F}}(L(a, b))$

$$
\simeq \begin{cases}V\left(\varpi_{b-a+1}\right)_{(-1)^{b-a} q_{t} q^{a+b-2}} & \text { for } 1 \leq a \leq b \leq N-2, \quad b-a+1<n, \\ V\left(\varpi_{n}\right)_{q^{2 b}} \nabla V\left(\varpi_{n}\right)_{q^{2 a+N-3}} & \text { for } 1 \leq a \leq b \leq N-2, \quad b-a+1 \geq n, \\ V\left(\varpi_{n}\right)_{q^{2 a+N-3}} \nabla V\left(\varpi_{n}\right)_{q^{2 b-2}} & \text { for } 1 \leq a \leq N-1<b, \quad b-a+1 \leq n, \\ V\left(\varpi_{N-b+a-1)}\right)_{(-1)^{b-a} q_{t} q^{a+b-3}} & \text { for } 1 \leq a \leq N-1<b, \quad b-a+1>n\end{cases}
$$

Proof. (i) and (ii) follow from Lemma 2.4.1.

(iii) Let $1 \leq a \leq b \leq N-2$. Then we have a homomorphism

$$
\widetilde{\mathcal{F}}(L(a, b)) \otimes \widetilde{\mathcal{F}}(L(b+1, N-1)) \rightarrow \widetilde{\mathcal{F}}(L(a, N-1)) \simeq V\left(\varpi_{n}\right)_{q^{2 a+N-3}} .
$$

Since $V\left(\varpi_{n}\right)_{q^{2 b}}$ is a left dual of $V\left(\varpi_{n}\right)_{q^{2 b+N-1}} \simeq \widetilde{\mathcal{F}}(L(b+1, N-1))$, we have

$$
\widetilde{\mathcal{F}}(L(a, b)) \longmapsto V\left(\varpi_{n}\right)_{q^{2 a+N-3}} \otimes V\left(\varpi_{n}\right)_{q^{2 b}} .
$$


It follows that

$$
\widetilde{\mathcal{F}}(L(a, b)) \simeq V\left(\varpi_{n}\right)_{q^{2 b}} \nabla V\left(\varpi_{n}\right)_{q^{2 a+N-3}}
$$

If $b-a+1<n$, then $2 b \leq 2 a+N-3$ and hence by (2.7), we have

$$
V\left(\varpi_{n}\right)_{q^{2 b}} \nabla V\left(\varpi_{n}\right)_{q^{2 a+N-3}} \simeq V\left(\varpi_{b-a+1}\right)_{(-1)^{b-a} q_{t} q^{a+b-2}} .
$$

By a similar way, we can obtain the case when $1 \leq a \leq N-1<b$.

Let us denote by $V^{(t)}\left(\varpi_{i}\right)_{x}$ the fundamental representation corresponding to $(i, x) \in$ $\mathscr{S}_{0}\left(A_{2 n-1}^{(t)}\right)(t=1,2)$. Combining (3.2), (3.3) and Proposition 3.2.1, we get the following

Corollary 3.2.2. Let $1 \leq i \leq n$ and let $p \in \mathbb{Z}$ satisfy $p \equiv i+1 \bmod 2$.

(i) If $i-1 \leq p \leq 2 N+i-3$, then we have

$$
\begin{aligned}
\phi_{1}\left(\left[V^{(1)}\left(\varpi_{i}\right)_{(-q)^{p}}\right]\right)=\phi_{2}\left(\left[V^{(2)}\left(\varpi_{i}\right)_{(-q)^{p}}\right]\right) & \\
= & \begin{array}{ll}
{\left[V\left(\varpi_{n}\right)_{q^{2 p}}\right]} & \text { for } p=i-1, \\
{\left[V\left(\varpi_{i}\right)_{(-1)^{i-1}} q_{t} q^{p-2}\right]} & \text { for } i+1 \leq p \leq 2 N-i-3, i<n, \\
{\left[V\left(\varpi_{n}\right)_{q^{p+n-1}} \nabla V\left(\varpi_{n}\right)_{q^{p+n-2}}\right]} & \text { for } i+1 \leq p \leq 2 N-i-3, i=n, \\
{\left[V\left(\varpi_{n}\right)_{q^{2 p-N-1}}\right]} & \text { for } p=2 N-i-1, \\
{\left[V\left(\varpi_{n}\right)_{q^{p+N-i-2}} \nabla V\left(\varpi_{n}\right)_{q^{p+i-3}}\right]} & \text { for } 2 N-i+1 \leq p \leq 2 N+i-3 .
\end{array}
\end{aligned}
$$

(ii) If $N-i-1 \leq p \leq 3 N-i-3$, then we have

$$
\begin{aligned}
\phi_{1}\left(\left[V^{(1)}\left(\varpi_{N-i}\right)_{(-q)^{p}}\right]\right)=\phi_{2}\left(\left[V^{(2)}\left(\varpi_{i}\right)_{-(-q)^{p}}\right]\right) \\
= \begin{cases}{\left[V\left(\varpi_{n}\right)_{q^{2 p}}\right]} & \text { for } p=N-i-1, \\
{\left[V\left(\varpi_{n}\right)_{q^{p+N-i-1}} \nabla V\left(\varpi_{n}\right)_{q^{p+i-2}}\right]} & \text { for } N-i+1 \leq p \leq N+i-3, \\
{\left[V\left(\varpi_{n}\right)_{q^{2 p-N-1}}\right]} & \text { for } p=N+i-1, \\
{\left[V\left(\varpi_{i}\right)_{\left.(-1)^{i-1} q_{t} q^{p-3}\right]}\right]} & \text { for } N+i+1 \leq p \leq 3 N-i-3, i<n, \\
{\left[V\left(\varpi_{n}\right)_{q^{p+n-2}} \nabla V\left(\varpi_{n}\right)_{q^{p+n-3}}\right]} & \text { for } N+i+1 \leq p \leq 3 N-i-3, i=n .\end{cases}
\end{aligned}
$$

Note that we have

$$
\phi_{t}\left(\left[V^{(t)}\left(\varpi_{i}\right)_{x q^{2 N}}\right]\right)=\left(\phi_{t}\left(\left[V^{(t)}\left(\varpi_{i}\right)_{x}\right]\right)\right)_{q^{2 N-2}}
$$

for all $(i, x) \in \mathscr{S}_{0}\left(A_{2 n-1}^{(t)}\right)(t=1,2)$. Hence the above corollary determines the maps $\phi_{t}$ $(t=1,2)$ on the set of fundamental representations.

\section{REFERENCES}

[1] T. Akasaka and M. Kashiwara, Finite-dimensional representations of quantum affine algebras, Publ. RIMS. Kyoto Univ., 33 (1997), 839-867.

[2] V. Chari and A. Pressley, Quantum affine algebras and their representations, in Representations of groups (Banff, AB, 1994), CMS Conf. Proc., 16, Amer. Math. Soc., Providence, RI, 1995, $59-78$.

[3] _ Quantum affine algebras and affine Hecke algebras, Pacific J. Math. 174 (2) (1996), 295-326.

[4] I. V. Cherednik, A new interpretation of Gelfand-Tzetlin bases, Duke Math. J., 54 (1987), 563577. 
[5] E. Frenkel and D. Hernandez, Langlands duality for finite-dimensional representations of quantum affine algebras, Lett. Math. Phys. 96 (2011) 217-261.

[6] E. Frenkel and N. Yu. Reshetikhin, The q-characters of representations of quantum affine algebras and deformations of $W$-algebras, Recent developments in quantum affine algebras and related topics, Contemp. Math. 248 (1999), 163-205.

[7] V. Ginzburg, N. Reshetikhin and E. Vasserot, Quantum groups and flag varieties, A.M.S. Contemp. Math. 175 (1994), 101-130.

[8] D. Hernandez, Kirillov-Reshetikhin conjecture: the general case, Int. Math. Res. Not. 2010 (1) (2010), 149-193.

[9] D. Hernandez and B. Leclerc, Cluster algebras and quantum affine algebras, Duke Math. J. 154 (2010), 265-341

[10] V. Kac, Infinite dimensional Lie algebras, 3rd ed., Cambridge University Press, Cambridge, 1990.

[11] S.-J. Kang, M. Kashiwara and M. Kim, Symmetric quiver Hecke algebras and R-matrices of quantum affine algebras, arXiv:1304.0323 [math.RT], to appear in Invent. Math.

[12] _ Symmetric quiver Hecke algebras and R-matrices of quantum affine algebras II, Duke Math. J. 164 (2015), no. 8, 1549-1602.

[13] S.-J. Kang, M. Kashiwara, M. Kim and S.-j. Oh, Simplicity of heads and socles of tensor products, Compos. Math. 151 (2015), no. 2, 377-396.

[14] Symmetric quiver Hecke algebras and R-matrices of quantum affine algebras III, Proc. Lond. Math. Soc. 111 (2015), no. 2, 420-444.

[15] _ Symmetric quiver Hecke algebras and R-matrices of quantum affine algebras IV, Selecta Math. 22 (2016), no. 4, 1987-2015.

[16] _ Monoidal categorification of cluster algebras II, arXiv:1502.06714.

[17] S.-J. Kang and E. Park, Irreducible modules over Khovanov-Lauda-Rouquier algebras of type $A_{n}$ and semistandard tableaux, J. Algebra 339 (2011), 223-251.

[18] M. Kashiwara, On level zero representations of quantum affine algebras, Duke. Math. J. 112 (2002), 117-175.

[19] M. Kashiwara, and S.-j. Oh, Categorical relations between Langlands dual quantum affine algebras: Doubly laced types. arXiv:1705.07542 [math.RT].

[20] M. Kashiwara and E. Park, Affinizations and R-matrices for quiver Hecke algebras, arXiv:1505.03241.

[21] M. Khovanov and A. Lauda, A diagrammatic approach to categorification of quantum groups I, Represent. Theory 13 (2009), 309-347.

[22] H. Nakajima, Quiver varieties and t-analogue of q-characters of quantum affine algebras, Annals of Math. 160 (2004), 1057-1097.

[23] S.-j. Oh, The denominators of normalized R-matrices of types $A_{2 n-1}^{(2)}, A_{2 n}^{(2)}, B_{n}^{(1)}$ and $D_{n+1}^{(2)}$, Publ. RIMS Kyoto Univ. 51 (2015), 709-744.

[24] R. Rouquier, 2-Kac-Moody algebras, arXiv:0812.5023v1 [math.RT].

[25] M. Vazirani, Parameterizing Hecke algebra modules: Bernstein-Zelevinsky multisegments, Kleshchev multipartitions, and crystal graphs, Transform. Groups. 7 (2002), 267-303. 
(Masaki Kashiwara) Research Institute for Mathematical Sciences, Kyoto University, Kyoto 606-8502, Japan \& Korea Institute for Advanced Study, Seoul 02455, Korea

E-mail address, Masaki Kashiwara: masaki@kurims.kyoto-u.ac.jp

(Myungho Kim) Department of Mathematics, Kyung Hee University, Seoul 02447, KOREA

E-mail address, Myungho Kim: mkim@khu.ac.kr

(Se-jin Oh) Department of Mathematics, Ewha Womans University, Seoul 120-750, Korea

E-mail address, Se-jin Oh: sejin092@gmail.com 